TRANSACTIONS OF THE

AMERICAN MATHEMATICAL SOCIETY

Volume 355, Number 10, Pages 4241-4263

S 0002-9947(03)03327-0

Article electronically published on June 18, 2003

\title{
THE GEOMETRY OF 1-BASED MINIMAL TYPES
}

\author{
TRISTRAM DE PIRO AND BYUNGHAN KIM
}

\begin{abstract}
In this paper, we study the geometry of a (nontrivial) 1-based $S U$ rank-1 complete type. We show that if the (localized, resp.) geometry of the type is modular, then the (localized, resp.) geometry is projective over a division ring. However, unlike the stable case, we construct a locally modular type that is not affine. For the general 1-based case, we prove that even if the geometry of the type itself is not projective over a division ring, it is when we consider a 2 -fold or 3-fold of the geometry altogether. In particular, it follows that in any $\omega$-categorical, nontrivial, 1-based theory, a vector space over a finite field is interpretable.
\end{abstract}

Geometric stability theory is one of the most important themes in model theory. Originally developing as a pure subject, it has turned out to be the major technical bridge connecting pure model theory and its applications to algebraic geometry and number theory. It mainly focuses on rank-1 types/structures or regular types where a canonical combinatorial geometry can be assigned. In other words, it is primarily concerned with geometric aspects of Shelah's stability theory [19], the study of stable structures. Arguably, the first major achievements of geometric stability theory are Zilber's results from the early 1980s (the translated version is [23) on a strongly minimal $\omega$-categorical structure. He showed that the geometry assigned to the structure is locally modular, hence, if nontrivial, must either be affine or projective over a finite field. A different proof was discovered independently by Cherlin, Harrington and Lachlan [6]. The results imply both the finiteness in rank of $\omega$-categorical, $\omega$-stable structures [6] and the non-finite axiomatizability of totally categorical theories [23, 6]. Since then, a number of leading researchers have obtained new enlightening results such as Buechler's dichotomy theorem [3], Hrushovski's group configuration theorems in several contexts [10, [11, his classification of a locally modular type [11, and other generalizations in the context of superstable theories [10, 11], [14]. Refined notions such as 1-basedness, regular types and $p$-weight have also been introduced. Pillay, in his book 17], makes a complete exposition of the subject. Hrushovski has now shifted the direction of his research towards applications in algebraic geometry and number theory, which has deepened and broadened the subject. It is well known that, using geometric stability theory and in particular Zilber's principle on "Zariski structures", he solved the Mordell-Lang conjecture 12] and other problems in number theory.

From the mid 1990s, after the initial papers [15], 16] of Kim and Pillay, simplicity theory, introduced by Shelah [18], has developed rapidly and extensively. Simplicity

Received by the editors January 4, 2002 and, in revised form, March 11, 2003.

2000 Mathematics Subject Classification. Primary 03C45.

The second author was supported by an NSF grant. 
theory is the study of a class of structures, called simple, properly containing that of stable structures. The central organizing principle has been the Independence Theorem (type amalgamation), leading to the introduction of Lascar strong types, canonical bases and hyperimaginaries. Definability and the interplay with local stability have also played an important developing role. An account on the subject has been written by Wagner 22, one of the major contributors. However, there are a number of obstacles to initiating an analogous geometric simplicity theory. This is mainly due to the lack of stationarity. Hrushovski's counterexample shows that Zilber's result on $\omega$-categorical strongly minimal structures cannot be generalized in the context of $\omega$-categorical rank-1 structures. Namely, there is a non-1-based, rank-1 $\omega$-categorical structure 13 .

In this paper we attempt to develop initial geometric simplicity theory using classical results in geometry as essential tools. We succeed in obtaining fruitful positive results and examples. We mainly study the solution set $D$ of a nontrivial 1-based (simple) $S U$ rank-1 Lascar strong type. Any finite tuple (not in the algebraic closure of the empty set) in a simple structure realizes a rank-1 Lascar strong type over some parameters. In particular, in any 1-based or supersimple theory, we can find a rank-1 type over a finite parameter. Moreover, if the 1-based theory is $\omega$-categorical, then the theory must have finite $S U$-rank (and is therefore supersimple). So every type is coordinatized by rank-1 types [9]. Hence studying such a 1-based $D$ is important in understanding $\omega$-categorical or even general 1-based theories. (As is well known, any $\omega$-categorical, $\omega$-stable theory is 1-based. The class of $\omega$-categorical 1-based structures properly contains that of smoothly approximable structures, too.) Furthermore, in the case of a supersimple rank-1 theory, since any model is the disjoint union of $\operatorname{acl}(\emptyset)$ and the solution sets of rank-1 Lascar strong types (over $\emptyset$ ), we can restrict our attention to the solution set of a Lascar strong type. In [21], using his notion of "generic pair", Vassiliev investigated the rank-1 theory. Here we study rank-1 types using a straightforward argument so that we can provide, for example, a direct proof of the fact that linearity implies 1-basedness. We also obtain the following more general results.

Local modularity of $D$ implies 1-basedness (however, the converse is not true in the general simple theory context, unlike the stable case). We first prove that

the (local, resp.) modularity of the pregeometry of $D$

implies that the (localized, resp.) geometry is isomorphic to a

projective geometry over a division ring.

These facts give the impression that, as in the stable case, the geometry of locally modular $D$ should be affine over a division ring. However, we construct an example refuting this surmise.

On the other hand, it follows from $(*)$ that if $D$ is (locally) modular and $\omega$ categorical, then, after possibly naming a point, a sort of $D^{e q}$ representing the geometry of $D$ has a (definitional) stable reduct preserving independence. The reduct must be strongly minimal, having a projective geometry over a finite field. Then, by the classical result on strongly minimal structures, a definable vector space over a finite field can be recovered inside the reduct. In particular, an infinite group is interpretable. For the more general case of a 1-based $\omega$-categorical $D$, we also show that a sort representing the geometry on some finite union of sorts in $D^{e q}$ has a generic (i.e., independence-preserving) strongly minimal stable reduct whose 
geometry is projective over a finite field. Again, a projective space over a finite field is interpretable. In fact, we obtain a more general result. Namely, for any 1-based $D$, we prove that the canonical geometry, extending (or completing) the geometry of $D$, on the set of canonical bases of surfaces in $D^{3}$, is projective over a division ring (Theorem 3.9) (assuming nontriviality). Here, by a surface in $D^{3}$, we mean a rank-2 Lascar strong type of a tuple in $D^{3}$. It follows that in any $\omega$-categorical, nontrivial, 1-based theory, a vector space over a finite field is interpretable over a finite parameter.

In this paper we assume that the underlying theory $T$ is simple, having, for convenience, elimination of hyperimaginaries. This assumption can be removed if $T$ is supersimple [4, or by working in $\bar{M}^{\text {heq }}$ and replacing notions in $\bar{M}^{e q}$ with corresponding notions in $\bar{M}^{\text {heq }}$ (e.g., $a c l$ by $b d d$, and so on).

\section{LOCALLY MODULAR TYPE}

We start this section by recalling the definitions of (pre)geometry, modularity and local modularity $([17,2.1])$.

Definition 1.1. Let $S$ be a set. If an operation $c l: \mathcal{P}(S) \rightarrow \mathcal{P}(S)$ satisfies the following properties, then we say that $(S, c l)$ is a pregeometry.

(1) For $A \subseteq S, A \subseteq \operatorname{cl}(A)=\operatorname{cl}(\operatorname{cl}(A))$.

(2) For $A \subseteq B \subseteq S, \operatorname{cl}(A) \subseteq \operatorname{cl}(B)$.

(3) For $A \subseteq S$ and $a, b \in S$, if $a \in \operatorname{cl}(A b) \backslash \operatorname{cl}(A)$, then $b \in \operatorname{cl}(A a)$.

(4) If $a \in \operatorname{cl}(A)$, then $a \in \operatorname{cl}\left(A_{0}\right)$, for some finite $A_{0} \subseteq A$.

The pregeometry $(S, c l)$ is said to be homogeneous if for any closure $X$ of a finite set and $a, b \in S \backslash X$, there is an automorphism of $(S, c l)$ that fixes $X$ pointwise and moves $a$ to $b$. We say that the pregeometry $(S, c l)$ is a geometry if $c l(\emptyset)=\emptyset$ and $\operatorname{cl}(a)=\{a\}$ for all $a \in S$. If $(S, c l)$ is a pregeometry, then we can associate a canonical geometry $(\hat{S}, \hat{c l})$, where $\hat{S}=\{c l(a) \mid a \in S \backslash \operatorname{cl}(\emptyset)\}$ and for $A \subseteq S$ we put $\hat{A}=\{c l(a) \mid a \in A\}$ and $\hat{c l}(\hat{A})=\{c l(b) \mid b \in \operatorname{cl}(A)\}$.

Let $(S, c l)$ be a pregeometry. We say that $A(\subseteq S)$ is independent if $a \notin$ $\operatorname{cl}\left((A \backslash\{a\})\right.$ for all $a \in A$. Given $B$, a subset $B_{0} \subseteq B$ is said to be a basis for $B$ if $B \subseteq \operatorname{cl}\left(B_{0}\right)$ and $B_{0}$ is independent. It follows that any two bases for $B$ have the same cardinality, denoted by $\operatorname{dim}(B)$. Any $A \subseteq S$ gives a localized pregeometry on $S$ defined by $\operatorname{cl}_{A}(B)=\operatorname{cl}(A \cup B)$, and a notion of dimension over $A(\operatorname{dim}(-/ A))$. For $A, B, C \subseteq S$, if $\operatorname{dim}\left(A^{\prime} / C\right)=\operatorname{dim}\left(A^{\prime} / B \cup C\right)$ for any finite $A^{\prime} \subseteq A$, then we say that $A$ is independent from $B$ over $C$.

Definition 1.2. Let $(S, c l)$ be a pregeometry.

(1) $(S, c l)$ is trivial if $\operatorname{cl}(A)=\bigcup\{c l(\{a\}): a \in A\}$ for all $A \subseteq S$.

(2) $(S, c l)$ is modular if $X$ is independent from $Y$ over $X \cap Y$ for all closed $X, Y$, or equivalently, if $\operatorname{dim}(X)+\operatorname{dim}(Y)=\operatorname{dim}(X \cup Y)+\operatorname{dim}(X \cap Y)$ for finite dimensional closed $X$ and $Y$.

(3) $(S, c l)$ is locally modular if it is modular over some point in $S$.

(4) $(S, c l)$ is locally finite if the closure of a finite set is finite.

(5) A 3-tuple $\left(c a_{1} a_{2}\right) \in S$ is a non-modular triangle if $\operatorname{dim}\left(c a_{1} a_{2}\right)=3, \operatorname{cl}\left(a_{1} a_{2}\right)$ $=\operatorname{cl}\left(a_{1}\right) \cup \operatorname{cl}\left(a_{2}\right)$ and there are $u_{i} \in \operatorname{cl}\left(\operatorname{ca} a_{i}\right) \backslash\left(\operatorname{cl}(c) \cup \operatorname{cl}\left(a_{i}\right)\right)$ for $i=1,2$. 
Let $S^{\prime}$ be the geometry of a pregeometry $S ; S$ is trivial (modular, or locally modular, resp.) if and only if $S^{\prime}$ is (resp.). Moreover, each of the properties is preserved under localization. Thus modularity implies local modularity.

Fact 1.3. $(S, c l)$ is modular iff for any subset $A$ and $b \in S$, whenever $c \in \operatorname{cl}(A b)$, then $c \in c l(a b)$ for some $a \in \operatorname{cl}(A)$.

Remark 1.4. (1) Suppose that $(S, c l)$ has a non-modular triangle $\left(c a_{1} a_{2}\right)$. Then $(S, c l)$ is not modular: Let $u_{i}$ be as in 1.2.5. First, suppose $\operatorname{cl}\left(u_{1}\right)=\operatorname{cl}\left(u_{2}\right)$. Since $a_{1}, a_{2} \in \operatorname{cl}\left(c u_{1}\right) \cup \operatorname{cl}\left(c u_{2}\right)=\operatorname{cl}\left(c u_{1}\right)$, we get $2=\operatorname{dim}\left(c u_{1}\right)=\operatorname{dim}\left(c a_{1} a_{2} u_{1}\right)=$ 3 , a contradiction. Hence $\operatorname{dim}\left(u_{1} u_{2}\right)=2$. Now $a_{1} \notin \operatorname{cl}\left(u_{1} u_{2}\right)$, as otherwise, since $c \in \operatorname{cl}\left(a_{1} u_{1} u_{2}\right)=\operatorname{cl}\left(u_{1} u_{2}\right)$ and $a_{2} \in \operatorname{cl}\left(c u_{2}\right)$, we would have $\operatorname{dim}\left(u_{1} u_{2}\right)=$ $\operatorname{dim}\left(c a_{1} a_{2} u_{1} u_{2}\right)=3$, a contradiction. Similarly, $a_{2} \notin \operatorname{cl}\left(u_{1} u_{2}\right)$. Recall that $\operatorname{cl}\left(a_{1} a_{2}\right)=\operatorname{cl}\left(a_{1}\right) \cup \operatorname{cl}\left(a_{2}\right)$. Hence $\operatorname{cl}\left(a_{1} a_{2}\right) \cap \operatorname{cl}\left(u_{1} u_{2}\right)=\operatorname{cl}(\emptyset)$. Thus

$$
4=\operatorname{dim}\left(a_{1} a_{2}\right)+\operatorname{dim}\left(u_{1} u_{2}\right) \neq \operatorname{dim}\left(a_{1} a_{2} u_{1} u_{2}\right)+\operatorname{dim}\left(\operatorname{cl}\left(a_{1} a_{2}\right) \cap \operatorname{cl}\left(u_{1} u_{2}\right)\right)=3 .
$$

This shows that $S$ is not modular.

(2) If $(S, c l)$ is a modular geometry and $c l(c d)=\{c, d\}$ for any two distinct points $c, d \in S$, then $S$ is trivial: By (4) of 1.1, it is sufficient to show that the closure of any finite set is itself. Suppose not, say there exists $d \notin A=\left\{a_{1}, \ldots, a_{n}\right\}$ such that $d \in$ $\operatorname{cl}(A)$. Then $\operatorname{cl}\left(a_{n} d\right)=\left\{a_{n}, d\right\}$ and $\operatorname{cl}\left(a_{1} \ldots a_{n-1}\right)=\left\{a_{1}, \ldots, a_{n-1}\right\}$ by the induction hypothesis. By modularity $n=\operatorname{dim}\left(a_{1} \ldots a_{n}\right)=\operatorname{dim}\left(a_{1} \ldots a_{n-1}\right)+\operatorname{dim}\left(a_{n} d\right)=n+1$, a contradiction.

Let us first state two important classical results on geometries, which will be used later.

Fact 1.5 ([1]). A projective (i.e., nontrivial and modular) geometry of dimension $\geq 4$ in which each closed set of dimension 2 contains at least 3 elements is isomorphic to a projective geometry over a division ring.

Fact 1.6 ([7]). A locally projective (i.e., nontrivial and locally modular), locally finite geometry of dimension $>4$, in which all closed sets of dimension 2 have the same size, is an affine or projective geometry over a finite field.

(For some reason, in the above Fact 1.6, the dimension condition is erroneously known to be $\geq 4$ to model theorists. This may be due to the fact that, in Doyen and Hubaut's original paper, they counted their dimension 1 less than what is commonly defined by model theorists. They even constructed a counterexample in dimension 4.)

From now on, we consider a pregeometry given on a type. As usual, the saturated model $\bar{M}$ of the simple theory $T$ is fixed. We work in $\bar{M}^{e q}$. (Tuples and sets are small from $\bar{M}^{e q}$.) We also fix a set $D$, which is a solution set (in the saturated model) of a Lascar strong type. For notational simplicity, we suppose $D$ is over $\emptyset$. Let us further suppose that $(D, c l)$ forms a pregeometry, where $c l$ is an $A u t(\bar{M})$ invariant closure relation on $D$ (i.e., $a \in \operatorname{cl}(B)$ if and only if $f(a) \in \operatorname{cl}(f(B))$ for each $f \in \operatorname{Aut}(\bar{M}))$. For safety, we assume that $\operatorname{dim}(D)=\operatorname{Card}(\bar{M})$. The typical examples of $D$ are, of course, rank-1 types with $\operatorname{cl}(-)=\operatorname{acl}(-) \cap D$, or regular types. $D^{\prime}$ denotes the geometry of $D$. We shall use the same closure notation for the pregeometry and its geometry.

Remark 1.7. The closure of $\emptyset$ is $\emptyset$. The size of $c l(p)$ does not depend on $p \in D$; hence $\left(D^{\prime}, c l\right)$ is homogeneous iff $(D, c l)$ is. 
Now, we observe that a modular $(D, c l)$ is well behaved 1

Lemma 1.8 ( $D$ modular). Suppose there exist $a, b \in D$ such that $\operatorname{dim}\{a, b\}=2$ and $\operatorname{cl}(a b)=\operatorname{cl}(a) \cup \operatorname{cl}(b)$. Then $D$ is trivial and homogeneous.

Proof. It suffices to show triviality. Moreover, we can assume that $D$ forms a geometry. Then the condition in the lemma simply says that $\operatorname{cl}(a b)=\{a, b\}$.

Now, by Remark 1.4.2, it is enough to show that $c l(c d)=\{c, d\}$ for any two distinct points $c, d$ : Suppose not, say there is a third point $u^{\prime} \in \operatorname{cl}(c d)$. Let $q(x, d)=$ $t p(c / d)$. Since $D$ is the solution set of a Lascar strong type, by the Independence Theorem for Lascar strong types, we can amalgamate $q(x, a)$ and $q(x, b)$. Let $c^{\prime}$ be a realization of a common nonforking extension of $q(x, a)$ and $q(x, b)$. Then $\operatorname{dim}\left(a, b, c^{\prime}\right)=3$, and there are $u, v$ such that $t p\left(c d u^{\prime}\right)=t p\left(c^{\prime} b u\right)=t p\left(c^{\prime} a v\right)$. Hence the tuple $\left(c^{\prime} a b\right)$ forms a non-modular triangle. By Remark 1.4.1, this contradicts the modularity of $D$ and finishes the proof.

In the above lemma, the assumption that $D$ is modular is necessary. We shall see in section 2 that even local modularity of $D$ is insufficient to imply the conclusion. There are other 1-based (3.7) and even a non-1-based example [13, where the closure of some pair of points can be 2 or 3 elements.

By now, we have the following theorems, due to Fact 1.5 and the previous lemma.

Theorem 1.9. If $D$ is modular, then either $D$ is trivial, or the geometry $D^{\prime}$ of $D$ is projective over a division ring. In either case, $(D, c l)$ is homogeneous.

Suppose that $D$ is $\omega$-categorical. Then $D^{\prime}$ is a sort in $D^{e q}$. Let $R_{n}$ be an $(n+1)$ ary relation on $D^{\prime}$ such that $\left(a_{0}, a_{1}, \ldots, a_{n}\right) \in R_{n}$ if and only if $a_{0} \in \operatorname{cl}\left(a_{1}, \ldots, a_{n}\right)$. Then $R_{n}$ is $\emptyset$-definable in $D^{\prime}$ (so in $D^{e q}$ ), and $\left(D^{\prime}, R_{n}\right)_{n \in \omega}$ is a (definitional) reduct of $D^{\prime}$. Hence, if $(D, c l)$ is nontrivial and modular, then by the previous theorem, $\left(D^{\prime}, R_{n}\right)_{n \in \omega}$ is strongly minimal and quantifier eliminable, with canonical pregeometry $\left(D^{\prime}, c l\right)$ that is projective over a finite field $F$. Then by the general result on strongly minimal structures, a vector space $(V,+, \lambda)_{\lambda \in F}$ over $F$ is definable in $\left(D^{\prime}, R_{n}\right)_{n \in \omega}^{e q}$, possibly over a finite parameter. We summarize this in the following theorem.

Theorem 1.10. Let $D$ be modular and $\omega$-categorical. Then the sort $S_{E}$ in $D^{e q}$, where $E(x, y) \equiv x \in c l(y)$, has a (definitional) stable reduct-preserving independence. Hence, in a nontrivial $D$, a vector space over a finite field is interpretable (i.e., definable in $D^{e q}$ over some finite set). In particular, the infinite additive group is definable in $D^{e q}$.

If, additionally, $D$ has $S U$-rank 1 and the usual algebraic closure relation, then $D$ itself has a generic stable reduct.

Now we investigate the locally modular case. We fix some notation. Choose $p \in D$, and let $D_{0}$ denote the pregeometry $D \backslash c l(p)$ with closure operation $c l_{p}$. $D_{0}^{\prime}$ denotes the geometry of $D_{0}$. Recall that we use the same closure notation for the pregeometry and its geometry. Note that $x \in \operatorname{cl}_{p}(y)$ is an equivalence relation on $D_{0}$. We obtain the following results $(1.11,12)$ analogous to the previous ones $(1.8,9)$. However, $(1.11,12)$ are not automatic consequences of $(1.8,9)$, since in the simple context $D_{0}$ need not be the solution set of a unique type over $p$.

\footnotetext{
${ }^{1}$ There may exist a reasonable demand for distinguishing between the terminology "modular type" and "type having a modular geometry". Here, we consider the two terms to be equal.
} 
For the rest of this section, $D$ is locally modular.

Lemma 1.11. Suppose that there are distinct $a^{\prime}, b^{\prime} \in D_{0}^{\prime}$ such that $c l_{p}\left(a^{\prime} b^{\prime}\right)=$ $\left\{a^{\prime} b^{\prime}\right\}$. Then $c l\left(c^{\prime} d^{\prime}\right)=\left\{c^{\prime}, d^{\prime}\right\}$ for any two points $c^{\prime}, d^{\prime}$ in $D_{0}^{\prime}$. Hence, $D_{0}^{\prime}$ is trivial.

Proof. Triviality will follow from the main assertion by Remark 1.4.2. Fix points $a, b$ in $D$ from $a^{\prime}, b^{\prime}$, respectively. Due to the limitation on type amalgamation, we need more complicated arguments to prove the lemma. We shall calculate dimension in $D$, unless stated otherwise.

Suppose that the lemma is not true. Then there is $u^{\prime} \in D_{0}^{\prime}$ such that $u^{\prime} \in$ $c l_{p}\left(c^{\prime} d^{\prime}\right) \backslash\left\{c^{\prime}, d^{\prime}\right\}$. Now choose (in $\left.D\right)$ points $c, d, u$ from $c^{\prime}, d^{\prime}, u^{\prime}$ respectively. Then clearly $\operatorname{dim}(p c d u)=3$, and

$$
\operatorname{dim}(p X)=3 \text { for each 2-subset } X \text { of }\{c, d, u\} .
$$

Case I. $u \in c l(c d)$. Then

(**) the closure of any two of the $u, c, d$ contains the third point.

Now, we claim the following.

Claim. There are $v_{i} \in D$ with $v_{i} \in v_{i}^{\prime} \in D_{0}^{\prime}$ (for $\left.i=1,2,3\right)$ such that $t p(c d u)=$ $t p\left(v_{1} b v_{2}\right)=t p\left(v_{1} a v_{3}\right)$, and $\operatorname{dim}\left(p a b v_{1}\right)=4$.

Proof of claim. Let $q(x, d)=t p(c / d)$. Then we can amalgamate $q(x, b)$ and $q(x, a)$. Let $v_{1}$ be a realization of a common nonforking extension. Then clearly there are $v_{2}, v_{3}$ such that $t p(c d u)=t p\left(v_{1} b v_{2}\right)=t p\left(v_{1} a v_{3}\right)$. Moreover, we can assume

$$
v_{1} \underset{a b}{\downarrow} p
$$

so that $\operatorname{dim}\left(p a b v_{1}\right)=4$. In particular, $p \notin \operatorname{cl}\left(v_{1} b\right)$. Now by $(* *), v_{2} \in \operatorname{cl}\left(v_{1} b\right)$. Hence $\operatorname{cl}(p) \neq \operatorname{cl}\left(v_{2}\right)$, and $v_{2}^{\prime} \in D_{0}$. Similarly $v_{3}^{\prime} \in D_{0}$. Therefore the claim is proved.

Since $u \in \operatorname{cl}(c d) \backslash\{c, d\}$, we have $v_{3} \in \operatorname{cl}(v, a) \backslash\{v, a\}$ and $v_{2} \in \operatorname{cl}(v, b) \backslash\{v, b\}$. If $v_{3} \in \operatorname{cl}(a p)$, then $p \in \operatorname{cl}\left(a v_{3}\right) \subseteq \operatorname{cl}\left(a v_{1}\right)$, contradicting $\operatorname{dim}\left(p a b v_{1}\right)=4$. Similarly, $v_{3} \notin \operatorname{cl}\left(v_{1} p\right)$ and $v_{2} \notin \operatorname{cl}(b p) \cup \operatorname{cl}\left(v_{1} p\right)$. Thus $\left(v_{1}^{\prime} a^{\prime} b^{\prime}\right)$ forms a non-modular triangle in $D_{0}^{\prime}$, contradicting modularity. Hence case I does not happen.

We consider the remaining case.

Case II. $u \notin c l(c d)$. Then by $(*)$,

$(* * *) \quad$ each 3 -element subset of $\{p, c, d, u\}$ has dimension 3.

This time, let $q(x, c d)=t p(p / c d)$. There are $u_{2}, u_{4}$ such that $t p(c d)=t p\left(a u_{2}\right)=$ $t p\left(b u_{4}\right)$ and $\operatorname{dim}\left(a b u_{2} u_{4}\right)=4$. Amalgamate $q\left(x, a u_{2}\right), q\left(x, b u_{4}\right)$ and let $u_{1} \in D$ be a realization of a common nonforking extension, independent of $p_{0}$. Then $\operatorname{dim}\left(p u_{1} a b u_{2} u_{4}\right)=6$. Now there are $u_{3} \in u_{3}^{\prime} \in D_{0}^{\prime}$ and $u_{5} \in u_{5}^{\prime} \in D_{0}^{\prime}$ such that $t p(p c d u)=t p\left(u_{1} a u_{2} u_{3}\right)=t p\left(u_{1} b u_{4} u_{5}\right)$. By use of $(* * *)$, one can see that $\operatorname{dim}\left(p u_{2} u_{3} u_{4} u_{5}\right)=5$ and $a^{\prime}, b^{\prime} \notin c l_{p}\left(u_{2}^{\prime} u_{3}^{\prime} u_{4}^{\prime} u_{5}^{\prime}\right)$. Thus $c l_{p}\left(a^{\prime} b^{\prime}\right) \cap c l_{p}\left(u_{2}^{\prime} u_{3}^{\prime} u_{4}^{\prime} u_{5}^{\prime}\right)=\emptyset$. This again violates the modularity of $D_{0}^{\prime}$. Hence Case II does not happen either.

Therefore Lemma 1.11 is proved.

Theorem 1.12. $D_{0}^{\prime}$ is either trivial or projective over a division ring.

Lemma 1.13. $D$ is trivial if and only if $D_{0}$ is trivial. 
Proof. We can safely assume that $D$ forms a geometry. It is enough to show that triviality of $D_{0}$ implies that of $D$. Let $D_{0}$ be trivial.

Claim. The closure of any two distinct points $\{p, q\}$ in $D$ is itself.

Proof of claim. Suppose not, say $r \in \operatorname{cl}(p q) \backslash\{p, q\}$. Choose

$$
s \notin c l(p q)=c l(p q r) .
$$

Since $D_{0}$ is trivial, $c l(p q s)=c l(p s) \cup \operatorname{cl}(p q)$ and $\operatorname{dim}(p q s)=3$. Hence $q, r \notin c l(p s)$. Now, since the localized pregeometry of $D$ at $s$ is equivalent to $D_{0}$ and $D_{0}$ is trivial, $c l(q s) \cup c l(p s)=c l(p q s)$. In particular, $r \in \operatorname{cl}(q s) \cup c l(p s)$. Hence, since $r \notin c l(p s)$, $r \in \operatorname{cl}(q s)$. Since $\operatorname{dim}(q s)=2, s \in \operatorname{cl}(q r)$, this contradicts $\left(^{*}\right)$. Thus the claim is verified.

Now, by the claim, for $n+1$ points $p, q_{1}, \ldots, q_{n}$, since $D_{0}$ is trivial, $c l\left(p q_{1} \ldots q_{n}\right)=$ $c l\left(p q_{1}\right) \cup \ldots \cup \operatorname{cl}\left(p q_{n}\right)=\left\{p, q_{1}, \ldots, q_{n}\right\}$. Hence $D$ is also trivial.

By the same argument as for Theorem 1.10 above, if $D$ is locally modular $\omega$ categorical, after naming a point $p$ in $D$, a sort in $\left(D_{p}\right)^{e q}$ representing $D_{0}^{\prime}$ has a generic (independence-preserving) stable reduct (over $p$ ). Hence, if $D$ is nontrivial, a vector space over a finite field is again interpretable over a finite parameter. We shall in fact prove the interpretability of a vector space in the more general 1-based context (3.23).

By now, one would naturally expect that, as in stable theories, a (nontrivial, non-modular) locally modular $D$ should be affine. However, the example in the following section shows that this is not the case.

\section{Non-AFFine LOCALly MODUlaR TYPE}

First, we fix an extended language $\mathcal{L}^{\prime}=\mathcal{L}_{\mathcal{A}} \cup\{P\}$ where $P$ is a unary predicate and $\mathcal{L}_{A}$ is the language of the theory of affine space over a finite field $F$ of cardinality at least 5 . (We shall see below a number of reasons why this condition is imposed. In fact, one can do the same construction with a projective space over a finite field of cardinality $\geq 4$.) Now, suppose $V_{1}$ is a finite affine space over $F$ generated by independent elements $\left\{x_{0}, \ldots, x_{n}\right\}$. Let $P$ colour some of the elements in $V_{1}$. We call the colouring good if for every line $l$ in $V_{1}$, we can find at least 2 points in $l$ coloured by $P$, and let $g c(n)>0$ be the finite number of good colourings, for $n \geq 1$, described by $\mathcal{L}^{\prime}$-formulas $\operatorname{Col}_{r, n}\left(x_{0}, x_{1}, \ldots, x_{n}\right)$. Now suppose $V_{2} \supset V_{1}$ is an affine space with basis $\left\{x_{0}, \ldots, x_{n+1}\right\}$. Then, given a good colouring $\operatorname{Col}_{r, n}\left(x_{0}, \ldots, x_{n}\right)$ of $V_{1}$, there exists a good colouring extending it to $V_{2}$. (For example, colour every point in $V_{2} \backslash V_{1}$ with $P$ while leaving the colouring on $V_{1}$. Since every line in $V_{2}$ either is already in $V_{1}$ or hits $V_{1}$ once, the condition $q \geq 5$ implies that this is a good colouring.) We denote the finite number of all possible such colourings by $g c(r, n)$ and let them be described by $\mathcal{L}^{\prime}$-formulas $\operatorname{Col}_{r, n}^{s, n+1}\left(x_{0}, x_{1}, \ldots, x_{n}, x_{n+1}\right)$, for $1 \leq s \leq g c(r, n)$. For $n=0$, we interpret $\operatorname{Col}_{0,0}^{s, 1}\left(x_{0}, x\right)$ and $\operatorname{Col}_{1,0}^{s, 1}\left(x_{0}, x\right)$ to mean that $P$ gives a good colouring on the line generated by $\left\{x_{0}, x\right\}$ extending the colourings $\neg P$ and $P$ respectively on $x_{0}$, and we let $g c(0,0)$ and $g c(1,0)$ denote the number of such colourings.

Now let $T_{A}$ denote the theory of affine space over $F$. Then the $\mathcal{L}_{A}$-formulas $x \in \operatorname{cl}\left(y_{1}, \ldots, y_{n}\right)$ and $\operatorname{dim}\left(y_{1} \ldots y_{n}\right)=m(\leq n)$ make sense. (We note here that the 
dimension defined throughout the paper is one more than the usual affine subspace dimension.) The theory $T_{L M}$ is given in the language $\mathcal{L}^{\prime}$ by the following axioms:

$$
T_{L M} \equiv T_{A} \cup\{L M\} \cup\left\{F_{n}: n \geq 0\right\},
$$

where $L M$ (local modularity) is the following sentence in $\mathcal{L}^{\prime}$ :

$$
L M \equiv \forall x \forall y\left(x \neq y \rightarrow \exists z_{1} \exists z_{2}\left(z_{1} \neq z_{2} \wedge P\left(z_{1}\right) \wedge P\left(z_{2}\right) \wedge z_{1}, z_{2} \in \operatorname{cl}(x, y)\right)\right),
$$

$F_{n}$ (colour extension), for $n \geq 1$, is the $\mathcal{L}^{\prime}$-sentence

$$
\begin{aligned}
F_{n} \equiv & \bigwedge_{1 \leq r \leq g c(n)} \forall y_{0} \ldots y_{n}\left(\operatorname{dim}\left(y_{0} \ldots y_{n}\right)=n+1 \wedge \operatorname{Col}_{r, n}\left(y_{0}, y_{1}, \ldots, y_{n}\right)\right. \\
& \left.\rightarrow \bigwedge_{1 \leq s \leq g c(r, n)} \exists x\left(\operatorname{dim}\left(y_{0} \ldots y_{n} x\right)=n+2 \wedge \operatorname{Col}_{r, n}^{s, n+1}\left(y_{0}, y_{1}, \ldots, y_{n}, x\right)\right)\right),
\end{aligned}
$$

and $F_{0}$ is the sentence

$$
\begin{aligned}
F_{0} \equiv \forall y(\neg P(y) \rightarrow & \left.\bigwedge_{1 \leq s \leq g c(0,0)} \exists x\left(\operatorname{dim}(y x)=2 \wedge \operatorname{Col}_{0,0}^{s, 1}(y, x)\right)\right) \\
& \wedge \forall y\left(P(y) \rightarrow \bigwedge_{1 \leq s \leq g c(1,0)} \exists x\left(\operatorname{dim} c l(y x)=2 \wedge \operatorname{Col}_{1,0}^{s, 1}(y, x)\right)\right) .
\end{aligned}
$$

We first show that the theory $T_{L M}$ is consistent. This can be done by a union of chains argument inside a model $V$ of $T_{A}$. We first point out the following on any geometry.

Remark 2.1. Suppose that $(S, c l)$ is a geometry. Let $\left\{A_{i} \in S \mid i \in I\right\}$ be independent over $B \subseteq S$, i.e., for finite $\bar{a}_{i} \in A_{i}, \operatorname{dim}\left(\bar{a}_{i} / B\right)=\operatorname{dim}\left(\bar{a}_{i} / B \cup \bigcup_{j \neq i} A_{j}\right)$. Then:

(1) $\operatorname{cl}(B)=\operatorname{cl}\left(B A_{i_{1}} \ldots A_{i_{n}}\right) \cap \operatorname{cl}\left(B A_{j_{1}} \ldots A_{j_{m}}\right)$ for $i_{1}, \ldots, i_{n}, j_{1}, \ldots j_{m}$ distinct.

(2) Each line in $\operatorname{cl}\left(B \cup \bigcup_{i} A_{i}\right)$ either already lies in one of the $\operatorname{cl}\left(B A_{i}\right)$, or hits $\bigcup_{i} c l\left(B A_{i}\right)$ at most twice.

Proof. The first statement clearly comes from the fact that

$$
\operatorname{dim}\left(\bar{a}_{i_{1}} \ldots \bar{a}_{i_{n}} / B\right)=\operatorname{dim}\left(\bar{a}_{i_{1}} \ldots \bar{a}_{i_{n}} / B \cup \bigcup_{j \neq i_{1}, \ldots, i_{n}} A_{j}\right) .
$$

For the second, assume that a line in $\operatorname{cl}\left(B \cup \bigcup_{i} A_{i}\right)$ hits $\bigcup_{i} \operatorname{cl}\left(B A_{i}\right) 3$ times, but does not lie in any $c l\left(B A_{i}\right)$. Let $\{x, y, z\}$ be the hit points. Then clearly $x \in \operatorname{cl}\left(B A_{k}\right), y \in \operatorname{cl}\left(B A_{m}\right) \backslash \operatorname{cl}\left(B A_{k}\right), z \in \operatorname{cl}\left(B A_{n}\right) \backslash\left(\operatorname{cl}\left(B A_{m}\right) \cup \operatorname{cl}\left(B A_{k}\right)\right)$ for distinct $k, m, n$, whereas $z \in \operatorname{cl}(x y)$ and $z \in \operatorname{cl}\left(B A_{n}\right) \cap \operatorname{cl}\left(B A_{k} A_{m}\right)$. But this contradicts (1).

Now we proceed in a series of steps.

Step 1. Choose a $y_{0} \in V$ and label $y_{0}$ by $P$. Now add independent elements $\left\{x_{1}, \ldots, x_{g c(1,0)}\right\}$ over $y_{0}$ and colour each $c l\left(y_{0} x_{i}\right)$ to witness $F_{0}$ for $y_{0}$. Let $W_{1}=$ $c l\left(y_{0}, x_{1}, \ldots, x_{g c(1,0)}\right)$. Now colour all points in $W_{1} \backslash \bigcup_{i} c l\left(y_{0} x_{i}\right)$ with $P$. Then $W_{1}$ satisfies $L M$; if a line in $W_{1}$ already lies in one of $\operatorname{cl}\left(y_{0} x_{i}\right)$, then we are done by construction. Otherwise, by the above remark (2), the assumption $\operatorname{Card}(F) \geq 5$ ensures that we can pick up at least 3 points on the line labelled with $P$.

Step 2. We now want to satisfy $F_{0}$ in $W_{1} \backslash\left\{y_{0}\right\}$. So we repeat the method of Step 1 for each point in $W_{1} \backslash\left\{y_{0}\right\}$. Namely, for $a \in W_{1} \backslash\left\{y_{0}\right\}$, find independent $\left\{z_{1}, \ldots, z_{g c(k, 0)}\right\}$ that are also independent from $W_{1}(k=0,1$ depends on whether $a \in \neg P$ or $a \in P)$. Then colour all the points in $\operatorname{cl}\left(W_{1} \cup\left\{z_{i}\right\}_{i}\right)$ with $P$, except 
$\bigcup_{i} \operatorname{cl}\left(a z_{i}\right)$ and $W_{1} . W_{1}$ is already coloured. Colour each $\operatorname{cl}\left(a z_{i}\right)$ to witness $F_{0}$ for $a$. Then, for the same reason as in Step 1, with Remark 2.1, $\operatorname{cl}\left(W_{1} \cup\left\{z_{i}\right\}_{i}\right)$ satisfies $L M$. Now for $b \in W_{1} \backslash\left\{y_{0}, a\right\}$, do the same thing over $c l\left(W_{1} \cup\left\{z_{i}\right\}_{i}\right)$, and so on. We then obtain a subspace $U_{0} \supset W_{1}$ such that $U_{0}$ satisfies $L M$ and, for all $x \in W_{1}$, $F_{0}$ is witnessed inside $U_{0}$.

Step 3. Now by modifying Steps 1 and 2 , find $U_{1}\left(\supset U_{0}\right)$ to make $F_{1}$ hold for $W_{1}$ inside $U_{1}$. Namely, for given distinct $x, y \in W_{1}$, choose independent points $\left\{w_{k}\right\}_{k}$ independent from $U_{0}$ to extend the good colouring on $\operatorname{cl}(x y)$ to all possible good colourings on distinct affine planes $\operatorname{cl}\left(x y w_{k}\right)$. (These colourings are compatible, because $\operatorname{cl}(x y)=U_{0} \cap \operatorname{cl}\left(x y w_{k}\right)=\operatorname{cl}\left(x y w_{i}\right) \cap \operatorname{cl}\left(x y w_{j}\right)($ for $i \neq j$ by Remark 2.1.1).) Then colour all the points in $\operatorname{cl}\left(U_{0} \cup\left\{w_{k}\right\}_{k}\right)$ other than $U_{0}$ and $\bigcup_{k} \operatorname{cl}\left(x y w_{k}\right)$ with $P$. Again, as in Step 1, the fact that $\operatorname{Card}(F) \geq 5$ and Remark 2.1.2 guarantee that $c l\left(U_{0} \cup\left\{w_{k}\right\}_{k}\right)$ satisfies $L M$. For the next line from $W_{1}$ other than $c l(x y)$, repeat the process over $\operatorname{cl}\left(U_{0} \cup\left\{w_{k}\right\}_{k}\right)$. By iterating this argument, we obtain a chain of spaces $W_{1} \subset U_{0} \subset \ldots \subset U_{n}$ so that $U_{n}$ witnesses $\left\{F_{0}, \ldots, F_{n}\right\}$ for $W_{1}$, and $U_{n}$ satisfies $L M$. We can choose $n$ large enough (depending on the dimension of $W_{1}$ ) so that in fact $U_{n}$ witnesses all $F_{i}$ (for $i \geq 0$ ) for $W_{1}$.

Rename $U_{n}$ as $W_{2}$, and repeat the previous steps for $W_{2}$. Continuing in this way, we obtain a chain of spaces $y_{0} \subset W_{1} \subset W_{2} \subset \ldots W_{i} \subset \ldots$ such that each $W_{i}$ satisfies $L M$ (by 2.1.2), and all $F_{i}(i \geq 0)$ are witnessed for $W_{i}$ inside $W_{i+1}$. Let $W=\bigcup_{i} W_{i}$; then by construction $W \models T_{A} \cup\{L M\} \cup\left\{F_{i}: i \geq 0\right\}$. Hence $T_{L M}$ is consistent.

We now have the following theorem.

Theorem 2.2. $T_{L M}$ is $\omega$-categorical, complete, and has quantifier elimination. Moreover, $T_{L M}$ is a model companion of $T_{A} \cup\{L M\}$.

The axioms $\left\{F_{n}: n \geq 0\right\}$ of colour extensions allow a partial isomorphism to be continued. Hence quantifier elimination follows. In particular, for an independent tuple $\left(a_{0}, \ldots, a_{n}\right), \operatorname{tp}\left(a_{0}, \ldots, a_{n}\right)$ is determined by the colouring on $\operatorname{cl}\left(a_{0}, a_{1}, \ldots, a_{n}\right)$. $\omega$-categoricity and hence completeness also follow by a straightforward back and forth partial isomorphism argument. Now, given a model $M$ of $T_{A} \cup\{L M\}$, by doing the above process starting with $M$, one can obtain an extension $N$ of $M$ which is a model of $T_{L M}$. Hence $T_{L M}$ is a model companion of $T_{A} \cup\{L M\}$.

We now want to show that $T_{L M}$ is a simple theory. As we observed, the condition $\operatorname{Card}(F) \geq 4$ is enough to obtain the previous theorem. However, we shall see that we need $\operatorname{Card}(F) \geq 5$ to ensure $T_{L M}$ is simple, particularly for the Independence Theorem. Let us first define a notion of independence on subsets $A, B, C$ of a saturated model $\bar{V}$ of $T_{L M}$ by

$$
A \underset{C}{\downarrow} B
$$

iff $A$ is independent from $B$ over $C$ in the sense of affine space (i.e., in $\mathcal{L}_{A}$ ). It follows easily that this notion is automorphism-invariant and satisfies symmetry, transitivity, finite character and local character. We need to check the extension axiom and the Independence Theorem. Below we keep using $c l$ to denote affine closure.

Nonforking extension. So let $\bar{a}$ and $B, C$ be a tuple and two small subsets of $\bar{V}$. Then clearly there is $\bar{a}^{\prime}$ such that $\bar{a}^{\prime}$ is $\left(\mathcal{L}_{A^{-}}\right)$independent from $C$ over $B$. Hence, as in 2.1.1, $\operatorname{cl}\left(\bar{a}^{\prime} B\right) \cap \operatorname{cl}(B C)=\operatorname{cl}(B)$. Therefore the colouring of $\operatorname{cl}\left(\bar{a}^{\prime} B\right)$ according 
to $\operatorname{cl}(\bar{a} B)$ is compatible with the colouring on $\operatorname{cl}(B C)$. Again colour all the points in $\operatorname{cl}\left(\bar{a}^{\prime} B C\right)$ with $P$ except $\operatorname{cl}\left(\bar{a}^{\prime} B\right)$ and $\operatorname{cl}(B C)$. By an argument similar to that in Remark 2.1.2 $\operatorname{cl}\left(\bar{a}^{\prime} B C\right)$ with the new colouring satisfies $L M$. Hence by the axioms $\left\{F_{n}\right\}$ we can embed $\bar{a}^{\prime}$, while fixing $B C$ with the new colouring, into $\bar{V}$, and then get the $\left(\mathcal{L}^{\prime}\right.$-)nonforking extension of $t p(\bar{a} / B)$ over $B C$.

The Independence Theorem for types. We will verify this over any closed set $B$. Here types are $\mathcal{L}^{\prime}$-types. Let $\bar{a}$ and $\bar{b}$ be independent tuples over $B$, and $\bar{c}, \bar{d}$ tuples such that

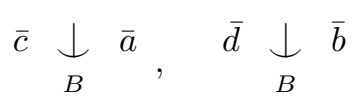

and $t p(\bar{c} / B)=t p(\bar{d} / B)$. By the extension property above, we can choose a tuple $\bar{e}$ independent from $\bar{b}$ over $B \bar{a}$ such that $\operatorname{tp}(\bar{c} / B \bar{a})=\operatorname{tp}(\bar{e} / B \bar{a})$. Then $\{\bar{e}, \bar{a}, \bar{b}\}$ is independent over $B$. Consider $\operatorname{cl}(B \bar{e} \bar{a} \bar{b})$. We now newly colour $\operatorname{cl}(B \bar{e} \bar{a} \bar{b})$ while leaving the (old) colouring on $\operatorname{cl}(B \bar{e} \bar{a}) \cup \operatorname{cl}(B \bar{a} \bar{b})$, as follows. First, colour $\operatorname{cl}(\bar{e} ; B \bar{b})$ according to $\operatorname{cl}(\bar{d} ; B \bar{b})$. Since $t p(\bar{e} / B)=t p(\bar{d} / B)$, on $\operatorname{cl}(B \bar{e} \bar{b}) \cap(\operatorname{cl}(B \bar{a} \bar{b}) \cup \operatorname{cl}(B \bar{a} \bar{e}))=$ $c l(B \bar{b}) \cup \operatorname{cl}(B \bar{e})$ (Remark 2.1.1), the two colourings are the same. Hence the new colouring is compatible with the colouring on $\operatorname{cl}(B \bar{e} \bar{a}) \cup \operatorname{cl}(B \bar{a} \bar{b})$. Then colour the rest of the points in $\operatorname{cl}(B \bar{e} \bar{a} \bar{b}) \backslash(\operatorname{cl}(B \bar{a} \bar{b}) \cup \operatorname{cl}(B \bar{e} \bar{a}) \cup \operatorname{cl}(B \bar{e} \bar{b}))$ with $P$. Now, since every line in $\operatorname{cl}(B \bar{e} \bar{a} \bar{b})$ either already lies in $\operatorname{cl}(B \bar{a} \bar{b}) \cup \operatorname{cl}(B \bar{e} \bar{a}) \cup \operatorname{cl}(B \bar{e} \bar{b})$ or hits the set at most 3 times, the condition $\operatorname{Card}(F) \geq 5$ ensures that this new colouring on $\operatorname{cl}(B \bar{e} \bar{a} \bar{b})$ is a good colouring. Since we can embed $\bar{e}$ with the new colouring on $\operatorname{cl}(B \bar{e} \bar{a} \bar{b})$ while fixing $B \bar{a} \bar{b}$, the Independence Theorem for types holds. (Hence the notion of type coincides with that of Lascar strong type.)

So $T_{L M}$ is simple. Moreover, in $\bar{V}\left(\models T_{L M}\right)$, since $\mathcal{L}^{\prime}$-independence is the same as $\mathcal{L}_{A}$-independence, $T_{L M}$ is an $S U$-rank-1 theory.

Theorem 2.3. $T_{L M}$ is simple with $S U$-rank 1 , having the same forking independence notion (and so algebraic relations) as $T_{A}$.

Now we take the solution set $\bar{V}(P)=\{a \in \bar{V} \mid P(a)\}$. Since we checked the Independence Theorem over any closed set, in particular over $\emptyset, \bar{V}(P)$ is a 1-(Lascar strong) type. We also have that $\bar{V}(P)$ is simple, rank-1 and $\omega$-categorical. It remains to show that $\bar{V}(P)$ is locally modular but not affine.

Local modularity. Choose $d \in \bar{V}(P)$. Let ef be a pair and $B$ a set from $\bar{V}(P)$ such that $\operatorname{dim}_{d}(e f)=2$ and $\operatorname{dim}_{d}(e f / B)=1$ inside $\bar{V}(P)$. Working in $\bar{V}$, we also have $\operatorname{dim}_{d}(e f)=2$ and $\operatorname{dim}_{d}(e f / B)=1$. Since the geometry of $\bar{V}$ is affine, $\operatorname{acl}(\operatorname{def}) \cap \operatorname{acl}(d B)=a c l(d g)$, where $g \neq d$ is in $\bar{V}$. By the axiom $L M$ for $\bar{V}$ we can assume that $g \in \bar{V}(P)$. Hence, $\operatorname{dim}_{d}\left(c l_{1}(\operatorname{def}) \cap c l_{1}(d B)\right)=1$, where $c l_{1}(-)=$ $\operatorname{acl}(-) \cap \bar{V}(P)$ is the closure inside $\bar{V}(P)$. So $\bar{V}(P)$ is a locally modular geometry.

Not affine. For this it is sufficient to observe that a good colouring of a line in $\bar{V}$ can label $r$ points where $2 \leq r \leq \operatorname{Card}(F)$.

So we have constructed a nontrivial, locally modular $\omega$-categorical rank-1 structure/type whose geometry is neither affine nor projective over a finite field. Another example of this kind can be constructed, as mentioned previously, by repeating the same process on any projective space over a finite field of cardinality $\geq 4$. (This is, in a sense, a necessary requirement. See 3.13.) As is well known, there is no such example in a stable structure, and also by 1.9 there is no example of a modular one. 


\section{1-BASED TYPE}

Let us recall some basic facts on 1-basedness. In this section, algebraic closure is taken over $\bar{M}^{e q}$. The following fact is folklore:

Fact 3.1 ( $T$ simple). The following are equivalent:

(1) $T$ is 1-based, i.e., any sets $A$ and $B$ are independent over $\operatorname{acl}(A) \cap \operatorname{acl}(B)$.

(2) For any tuple $\bar{c}$ and a set $A, \operatorname{cb}(\bar{c} / A) \subseteq \operatorname{acl}(\bar{c})$.

(3)

$$
A \underset{C}{\downarrow} B
$$

iff $\operatorname{acl}(A C) \cap \operatorname{acl}(B C)=\operatorname{acl}(C)$.

(4) For any indiscernible sequence $I=\left\langle\bar{c}_{i} \mid i \in \omega\right\rangle, I \backslash\left\{\bar{c}_{0}\right\}$ is a Morley sequence over $\bar{c}_{0}$.

Proof. We only sketch the proof. The equivalence of 1 and 2 is well known. In 3 , the implication from left to right is true for any simple theory, and the reverse implication is clearly equivalent to $1.4 \Rightarrow 2$ follows from the fact that $c b(\bar{c} / A)$ is in the algebraic (bounded) closure of a Morley sequence from $\operatorname{Lstp}(\bar{c} / A)$. We supply a rather detailed proof for $1 \Rightarrow 4$. Note first that 1 implies

$$
\bar{c}_{0} \underset{A}{\downarrow} \bar{c}_{1} \bar{c}_{2} \ldots
$$

where $A=\operatorname{acl}\left(\bar{c}_{0}\right) \cap \operatorname{acl}\left(\bar{c}_{1} \bar{c}_{2} \ldots\right)$. Then since

$(*)$ any indiscernible sequence over $B$

$$
\text { is indiscernible over } \operatorname{acl}(B) \text { (even over } b d d(B)) \text {, }
$$

it follows that $\operatorname{tp}\left(\bar{c}_{1} \bar{c}_{2} \ldots / \operatorname{acl}\left(\bar{c}_{0}\right)\right)=\operatorname{tp}\left(\bar{c}_{2} \bar{c}_{3} \ldots / a c l\left(\bar{c}_{0}\right)\right)$. Hence

$$
A=\operatorname{acl}\left(\bar{c}_{0}\right) \cap \operatorname{acl}\left(\bar{c}_{2} \bar{c}_{3} \ldots\right) \text {. }
$$

Again by $(*), \operatorname{tp}\left(\bar{c}_{0} / \operatorname{acl}\left(\bar{c}_{2} \bar{c}_{3} \ldots\right)\right)=\operatorname{tp}\left(\bar{c}_{1} / \operatorname{acl}\left(\bar{c}_{2} \bar{c}_{3} \ldots\right)\right)$, and then $A=\operatorname{acl}\left(\bar{c}_{1}\right) \cap$ $\operatorname{acl}\left(\bar{c}_{2} c_{3} ..\right)$. Then, using 1 again,

$$
\bar{c}_{1} \underset{A}{\downarrow} \bar{c}_{2} \bar{c}_{3} \cdots .
$$

From transitivity,

$$
\bar{c}_{1} \underset{\bar{c}_{0} A}{\downarrow} \bar{c}_{2} \bar{c}_{3} \ldots
$$

and

$$
\bar{c}_{1} \underset{\bar{c}_{0}}{\downarrow} \bar{c}_{2} \bar{c}_{3} \ldots
$$

since $A \subseteq \operatorname{acl}\left(\bar{c}_{0}\right)$. Thus 4 follows.

When, for example, a theory $T$ is 1-based of finite $S U$-rank (e.g., any 1-based $\omega$ categorical $T$; see the remark before 3.23 ), then, since every tuple can be "built up" (coordinatized) from rank-1 tuples, understanding the properties of rank-1 types is essential. This is the topic of the current section.

From now on, we further assume that $D$ is the solution set of a rank-1 Lascar strong type with the usual algebraic closure relation. We shall observe a strong connection between 1-basedness and modularity. We first note the following folklore, and supply a simple proof. 
Fact 3.2. If $D$ is locally modular, then $D$ is 1-based.

Proof (Remark). Suppose that $W_{0}, W_{1} \subseteq \operatorname{acl}(X) \cap \operatorname{acl}(Y)$ and

$$
X \underset{W_{0}}{\downarrow} Y
$$

and

$$
X \underset{W_{1}}{\downarrow} Y .
$$

Then $\operatorname{acl}\left(W_{0}\right)=\operatorname{acl}\left(W_{1}\right)$, since

$$
W_{1} \underset{W_{0}}{\downarrow} Y
$$

and

$$
W_{0} \underset{W_{1}}{\downarrow} Y
$$

(This remark will be used occasionally.)

Claim 1. Choose $p \in D$. For any $A \subseteq D^{e q}$, there is $B \subseteq D$ containing $p$ such that $A$ and $B$ are interalgebraic over $p$. Indeed, there is $A_{0} \subseteq D$ such that $A \subseteq d c l\left(A_{0}\right)$. Let $A_{1}(\subseteq D)$ realizing $t p\left(A_{0} / A p\right)$ be independent from $A_{0}$ over $A p$. Now, since $D$ is modular over $p$, if we let $B=\operatorname{acl}\left(A_{0} p\right) \cap \operatorname{acl}\left(A_{1} p\right) \cap D$, then $A_{0}, A_{1}$ are independent over $B(=B p)$. By the remark above, $\operatorname{acl}(A p)=\operatorname{acl}(B p)$. Hence the claim is proved.

Claim 2. Given $B_{0}, B_{1} \subseteq D^{e q}, B_{0}$ and $B_{1}$ are independent over $B_{2}=\operatorname{acl}\left(B_{0} p\right) \cap$ $\operatorname{acl}\left(B_{1} p\right)$ for given $p \in D$ (i.e., $D$ is 1-based over $p$ ).

Suppose not; say $B_{0}$ forks with $B_{1}$ over $B_{2}$. By Claim 1, there are $C_{0}, C_{1}, C_{2} \subseteq D$ containing $p$ such that $C_{i}$ is interalgebraic with $B_{i}$ over $p$. In particular, $C_{0}$ forks with $C_{1}$ over $C_{2}$. (Note that $p \in B_{2}, C_{2}$.) Now since $D$ is modular over $p$, there must exist $d \in \operatorname{acl}\left(C_{0} C_{2}\right) \cap \operatorname{acl}\left(C_{1} C_{2}\right) \cap D$ such that $d \notin \operatorname{acl}\left(C_{2}\right)=\operatorname{acl}\left(B_{2}\right)$. However, since $\operatorname{acl}\left(C_{0} C_{2}\right)=\operatorname{acl}\left(\operatorname{acl}\left(C_{0}\right) \cup \operatorname{acl}\left(C_{2}\right)\right)=\operatorname{acl}\left(B_{0} p B_{2}\right)=\operatorname{acl}\left(B_{0} B_{2}\right)$ and similarly $\operatorname{acl}\left(C_{1} C_{2}\right)=\operatorname{acl}\left(B_{1} B_{2}\right)$, it follows that

$$
d \in \operatorname{acl}\left(C_{0} C_{2}\right) \cap \operatorname{acl}\left(C_{1} C_{2}\right)=\operatorname{acl}\left(B_{0} B_{2}\right) \cap \operatorname{acl}\left(B_{1} B_{2}\right) \subseteq B_{2} .
$$

This is a contradiction. Hence Claim 2 is proved.

Now we can finish the proof of the fact. Let $I=\left\langle\bar{c}_{i} \mid i \in \omega\right\rangle$ be an indiscernible sequence from $D^{e q}$. If $q \in D$ is independent from $I$, then after a $\overline{c_{0}}$ conjugation we may assume that $I$ is indiscernible over $q$. Now by Claim 2 (with a similar proof to $3.1,1 \Rightarrow 4), I \backslash\left\{\bar{c}_{0}\right\}$ is a Morley sequence over $q \bar{c}_{0}$. Since $q$ is independent from $I$, by transitivity $I \backslash\left\{\bar{c}_{0}\right\}$ is Morley over $\bar{c}_{0}$. Hence, $D$ is 1-based by 3.1.

The converse of 3.2 is false in general. As in [21, one can easily construct a counterexample by adding a generic predicate $P(x)$ to a vector space over a finite field. In Remark 3.7, we will deal with this example again.

Definition 3.3. We use $G(D)$ to denote the collection of all rank-1 elements (over $\operatorname{dom}(D))$ in $D^{e q}$.

Clearly $D \subseteq G(D)$, and for any small set $A$ containing $\operatorname{dom}(D), G(D)_{A}$ forms a pregeometry with the usual algebraic closure relation over $A$. We recall the definition of linearity. 
Definition 3.4. For a set $A$ containing $\operatorname{dom}(D)$, we say that $D_{A}\left(G(D)_{A}\right.$, resp.) is linear if for any two points $a, b \in D(G(D)$, resp.) and parameters $B$ with $S U(a b / A B)=1$ we have $S U(C / A) \leq 1$, where $C=c b(a b / A B)$.

Remark 3.5. (1) When defining linearity above, it is safe to assume that the parameters $B$ form a subset of $D$ (or $G(D)$ ), since we can preserve the rank by taking a Morley sequence as the parameter.

(2) If a canonical base $C b(p)$ of $p$ (=Lstp of some tuple from $D^{e q}$ over parameters containing $\operatorname{dom}(D))$ has rank 1 , then we can find a singleton $c$ in $D^{e q}$ which can be considered as the canonical base up to interalgebraicity. Namely, since $T$ has elimination of hyperimaginaries, any hyperimaginary $e$ of ordinal $S U$-rank is interdefinable with a sequence $\left(e_{i}: i<\omega\right)$ of imaginaries, and there must be a subsequence such that $e \subset \operatorname{acl}\left(e_{1}, \ldots e_{n}\right)$. In this case, we can take $e=C b(p)$ and find an element $c$ in the sequence such that $S U(c)=1$ and $C b(p) \subseteq \operatorname{acl}(c)$. From now on, for this case $(S U(C b(p))=1)$, when we refer to a canonical case of $p$, we actually mean any such $c \in D^{e q}$. Since we are mainly interested in the geometry of $D$, as we shall see, this abuse of notation will not cause any problems. (If $T$ does not have elimination of hyperimaginaries, then $G(D)$ is the set of rank-1 hyperimaginaries living in $D^{\text {heq }}$.)

(3) We now single out an easy but useful argument in the proof of Claim 1 in 3.2. Let $D \subseteq F \subseteq G(D)$, and let $(F, \operatorname{cl}(-) \cap F)$ form a modular subpregeometry. Then for any $A \subseteq D^{e q}$, there is $B \subseteq F$ such that $A$ and $B$ are interalgebraic: For each $c \in D^{e q}$, there is a finite tuple $\bar{d} \in D$ such that $c \in \operatorname{dcl}(\bar{d})$. Hence, there is $A_{0} \subseteq D \subseteq F$ such that $A \subseteq \operatorname{dcl}\left(A_{0}\right)$. Let $A_{1}(\subseteq F)$ realizing $\operatorname{tp}\left(A_{0} / A\right)$ be independent from $A_{0}$ over $A$. Let $B=\operatorname{acl}\left(A_{0}\right) \cap \operatorname{acl}\left(A_{1}\right) \cap F$. Since $F$ is modular, $A_{0}$ and $A_{1}$ are independent over $B$. Then, by the remark in the proof of 3.2 , $\operatorname{acl}(B)=\operatorname{acl}(A)$.

Hence, if $a \in G(D)$, then there is a tuple $\bar{b}=\left(b_{1}, \ldots, b_{n}\right) \in F$ such that $a, \bar{b}$ are interalgebraic. In particular, $b_{1} \in \operatorname{acl}(a)$; so $a$ forks with $b_{1}$. Since $r k(a)=1$, $a \in \operatorname{acl}\left(b_{1}\right)$, i.e., $a, b_{1}$ are interalgebraic. This says that the canonical geometries of $F$ and $G$ are identical.

Theorem 3.6. The following are all equivalent.

(1) $D\left(D^{e q}\right)$ is 1-based.

(2) $D$ is linear.

(3) $G(D)$ is linear.

(4) $G(D)$ is modular.

(5) $G(D)_{A}$ is linear, for any (some) small $A$.

(6) $G(D)_{A}$ is modular, for any (some) small $A$.

Proof. The proof of $1 \Rightarrow 2$ is the same as the stable case. For completeness, we supply a proof. Let $D$ be 1-based. Suppose that $S U(\operatorname{Lstp}(a b / B))=1(a, b, B$ from $D)$. By 1-basedness, $C=c b(a b / B)$ is in $a c l(a b)$. Now,

$$
S U(a b C)=S U(a b / C)+S U(C)=1+S U(C)=S U(C / a b)+S U(a b) \leq 2 .
$$

Hence $S U(C) \leq 1$. Thus $D$ is linear.

$2 \Rightarrow 3$. Assume $D$ is linear. Now let $x, y \in G(D), B \subseteq G(D)$ and $S U(x y / B)=1$ while

$$
S U(x y)=2 .
$$


Let $C=c b(x y / B)$. Then

$$
C \subseteq \operatorname{acl}(B) \text { and } S U(x y / C)=1\left(\begin{array}{ccc}
x y & \downarrow & B \\
& C &
\end{array}\right) .
$$

We will show that $C \subseteq \operatorname{acl}(x y)$. Now $\{x, y\}$ is in the definable closure of a finite independent subset of $D$. In fact, we can find an independent subset $F \cup\{a, b\}$ of $D$ such that $\{x, y\}$ and $\{a, b\}$ are interalgebraic over $F$, and $F,\{x, y\}$ are independent. Then there is $F^{\prime} \subseteq D$ such that $F$ and $F^{\prime}$ realize the same type over $x y$, and

$$
F^{\prime} \underset{x y}{\downarrow} a b B F ;
$$

hence

$$
F^{\prime} \underset{\emptyset}{\downarrow} x y a b B F
$$

Now, since $F$ and $F^{\prime}$ realize the same type over $x y$, there is $\left\{a^{\prime} b^{\prime}\right\}$ such that $a b F$ and $a^{\prime} b^{\prime} F^{\prime}$ realize the same type over $x y$. Note that

$$
\operatorname{acl}\left(x y F^{\prime}\right)=\operatorname{acl}\left(a^{\prime} b^{\prime} F^{\prime}\right)
$$

Claim 1. $S U\left(a^{\prime} b^{\prime} / F^{\prime} B\right)=1$.

First we note that

$$
a^{\prime} b^{\prime} \underset{F^{\prime}}{\downarrow} B .
$$

(Otherwise, by (2) and (3), $\{x, y\}$ is independent from $B$, which contradicts (1).) Now if $S U\left(a^{\prime} b^{\prime} / F^{\prime} B\right)=0$, then by (3), $x, y \in \operatorname{acl}\left(F^{\prime} B\right)$, and then by (2), $x, y \in$ $\operatorname{acl}(B)$, again contradicting (1). Hence $S U\left(a^{\prime} b^{\prime} / F^{\prime} B\right)=1$. Claim 1 is proved. Now

Therefore, by Claim 1 and the linearity of $D, S U\left(a^{\prime} b^{\prime} / \operatorname{acl}\left(a^{\prime} b^{\prime}\right) \cap \operatorname{acl}\left(F^{\prime} B\right)\right)=1$.

$$
\operatorname{acl}\left(a^{\prime} b^{\prime}\right) \cap \operatorname{acl}\left(F^{\prime} B\right) \subseteq \operatorname{acl}\left(a^{\prime} b^{\prime} F^{\prime}\right) \cap \operatorname{acl}\left(F^{\prime} B\right) \subseteq \operatorname{acl}\left(F^{\prime} B\right) .
$$

Thus, by transitivity, Claim 1, and (3),

$$
S U\left(a^{\prime} b^{\prime} / W\right)=1 \text {, where } W=\operatorname{acl}\left(a^{\prime} b^{\prime} F^{\prime}\right) \cap \operatorname{acl}\left(F^{\prime} B\right)=\operatorname{acl}\left(x y F^{\prime}\right) \cap \operatorname{acl}\left(F^{\prime} B\right) .
$$

Claim 2. $S U(x y / W)=S U(x y / W C)=1(=S U(x y / C))$.

Since $F^{\prime} \subseteq W$, by (3) and (4),

$$
x y \underset{F^{\prime}}{\underset{X}{\not}} W .
$$

Hence $S U(x y / W)=0$ or 1 . But if $S U(x y / W)=0$ then by (2) $x y \in \operatorname{acl}(B)$, which is not the case. Thus $S U(x y / W)=1$. Similarly, since $S U(x y / C)=1$, if $S U(x y / W C) \neq 1$, then by $(\star)$ and $(4), x y \in \operatorname{acl}(W C) \subseteq \operatorname{acl}\left(C F^{\prime} B\right) \subseteq \operatorname{acl}\left(F^{\prime} B\right)$. Then by $(2), x y \in \operatorname{acl}(B)$. This contradicts (1) again. Hence $S U(x y / W C)=1$, and Claim 2 is proved.

Now since $\operatorname{Lstp}(x y / C)$ is a canonical type, by Claim $2, C \subseteq \operatorname{acl}(W)$. Therefore by (4), $C \subseteq \operatorname{acl}\left(x y F^{\prime}\right)$. Then $C \subseteq \operatorname{acl}(x y)$, since (2) with ( $\star$ ) says that

$$
C \underset{x y}{\downarrow} F^{\prime} .
$$

We have proved that $G(D)$ is linear. 
$3 \Rightarrow 6$ and 4 . Suppose that $G(D)$ is linear. Let $a, b \in G(D)$, and $S U(a b / A)=2$, whereas $S U(a b / A B)=1$ for $B \subseteq G(D)$. Now, there is a Morley sequence $I$ of $\operatorname{tp}(a b / A B)$ in $G(D)$ such that $S U(a b / A B)=S U(a b / A B I)=S U(a b / I)=1$. By linearity of $G(D), c \in a c l(a b) \cap \operatorname{acl}(I) \cap a c l(A B)$ where $c=c b(a b / I)=c b(a b / A B)$, and $S U(c)=1$. Hence $c \in G(D)$ by the previous Remark 3.5.2. Moreover, by the rank calculation of $S U(c a b / A)$ we have $S U(c / A)=1$, i.e., $c \notin \operatorname{acl}(A)$. Thus $G(D)_{A}$ is modular by 1.3 .

$4 \Rightarrow 1$. This proof will be similar to the proof of Fact 3.2. Suppose not, say there are $a, b \in D^{e q}$ that are dependent over $U=\operatorname{acl}(a) \cap \operatorname{acl}(b)$. Now since $G(D)$ is modular, by Remark 3.5.3, there are $a_{0}, b_{0}, U_{0} \subseteq G(D)$ that are interalgebraic with $a, b, U$ respectively. In particular, $a_{0}$ forks with $b_{0}$ over $U_{0}$. Then, again by modularity, there must exist $c_{0} \in \operatorname{acl}\left(a_{0} U_{0}\right) \cap \operatorname{acl}\left(b_{0} U_{0}\right) \cap G(D)$ such that $c_{0} \notin$ $\operatorname{acl}\left(U_{0}\right)=\operatorname{acl}(U)$. However, since $\operatorname{acl}\left(a_{0} U_{0}\right)=\operatorname{acl}\left(\operatorname{acl}\left(a_{0}\right) \cup \operatorname{acl}\left(U_{0}\right)\right)=\operatorname{acl}(a U)$ and similarly $\operatorname{acl}\left(b_{0} U_{0}\right)=\operatorname{acl}(b U)$, it follows that $c_{0} \in \operatorname{acl}(a U) \cap \operatorname{acl}(b U) \subseteq U$, a contradiction. Therefore, $4 \Rightarrow 1$ is proved.

$3 \Rightarrow 5$. Suppose that $G(D)$ is linear. Let us show that $G(D)_{A}$ is linear. Let $a, b \in G(D)$, and $S U(a b / B)=1$ for $B$ containing $A$. Now there is a Morley sequence $I$ of $t p(a b / B)$ in $G(D)$ such that $S U(a b / B)=S U(a b / B I)=S U(a b / I)=1$. Then by linearity of $G(D)$ we have $S U(c / A) \leq S U(c) \leq 1$ where $c=c b(a b / I)=c b(a b / B)$. Thus $G(D)_{A}$ is linear.

5 or $6 \Rightarrow 3$. The proof will be similar to [17 2.2.6 (i) $\Rightarrow$ (ii)]. Let $a, b \in G(D)$, $B \subseteq G(D)$ and $S U(a b / B)=1$. Let $c=c b(a b / B)$. If $S U(a b)=1$, i.e., $\{a, b\}$ is independent from $B$, then, since $c \in \operatorname{acl}(\emptyset)$, there is nothing to prove. Thus we can assume that $S U(a b)=2$. Now we can further assume that $a b B$ is independent from $A$. Hence, $S U(a b / B A)=1$, whereas $S U(a b / A)=2$. Then by modularity (or linearity) of $G(D)_{A}$, there is $d \in \operatorname{acl}(a b A) \cap \operatorname{acl}(A B)$ with $S U(d / A)=1$. Hence $d$ and $a b$ are dependent over $A$. Thus $S U(a b / A d)=1=S U(a b / A B)$. Therefore $c=$ $c b(a b / A B) \in a c l(A d)$, and since $S U(d / A)=1$, by a rank calculation $S U(c / A) \leq 1$. Since $c$ and $A$ are independent, $S U(c) \leq 1$.

Remark 3.7. (1) Similarly, $D$ is linear iff $D_{A}$ is linear for any (some) $A$. We note that the geometry of a nonforking extension of $D$ over $A$ is a mere subgeometry of $D_{A}$. However, one can still see the fact that linearity (1-basedness) is invariant via parallelism. Indeed, let $q$ be a nonforking extension of $D$ over $A$. It suffices to check that $D$ is linear when $q$ is linear. The proof is the same as $3.6(5) \Rightarrow(3)$ with the trick that when an independent pair $\left(a_{0}, a_{1}\right)$ is chosen from $D$, by amalgamation there is $\left(b_{0}, b_{1}\right)$ such that $t p\left(a_{0}, a_{1}\right)=t p\left(b_{0}, b_{1}\right)$ and each $b_{i}$ realizes $q$.

However, as is known, (local) modularity is not invariant via parallelism in general. (Frank O. Wagner was the first person to point this out.) Affine or projective space equipped with a binary random graph relation serves as an example, for a reason similar to that described in (2).

(2) Vassiliev introduced a notion of generic substructure $\bar{M}_{1}$ of $\bar{M}$ for a supersimple $S U$-rank-1 theory (the submodel $\bar{M}_{1}$ of $\bar{M}$ does not have to be a small submodel). From [21], it follows that when we consider $D$ as the universe of a model $\bar{M}, D$ is 1-based iff $D_{\bar{M}_{1}}$ is modular. (He also proved the equivalence of 1 and 2 in Theorem 3.6 using a generic pair argument.)

However, in general, even if $D$ is 1-based, $D_{A}$ need not be modular over any (small) set $A$. Indeed, let $V$ be a (saturated) affine space over a field, and let $P$ be a generic predicate on $V$, as in [5]. Then there are two 1-types over $\emptyset$ 
isolated by $P(x)$ and $\neg P(x)$, both of which have $S U$-rank 1. By the genericity of $P$, both 1-types are 1-based. $V$ is already non-modular over $\emptyset$. Now let $W$ be a nonempty small subspace of $V$ and consider a line $l$, spanned by $\{a, b\}$ in $V$, independent from $W$; so $\operatorname{dim}(l / W)=2$. Now let $l^{\prime}$, spanned by $\left\{a^{\prime} b^{\prime}\right\}$, be parallel to $l$ such that $\operatorname{dim}\left(l / W l^{\prime}\right)=1$. Then there is $c \in V \backslash W$ such that $\operatorname{acl}(\mathrm{cW})=\operatorname{acl}(l W) \cap \operatorname{acl}\left(l^{\prime} W\right)$. However, since $W$ is small, by genericity of $P$, we can find $l, l^{\prime}$ such that $\left\{a, b, a^{\prime}, b^{\prime}\right\} \subseteq P$, whereas $P \cap \operatorname{acl}(c W)=P \cap W$. Then clearly $P$ cannot be modular over $W$, and similarly for $\neg P$. (We noticed later that a similar example is described in 21].)

To conclude, there are examples of nontrivial, 1-based $D$ that are either not locally modular, or locally modular but neither affine nor projective (section 2) and examples of 1-based $D$ that are affine/projective but not stable (e.g. an affine space with a binary random graph relation).

Since triviality of a (pre)geometry implies modularity, we have the following lemma by Remark 3.5.3.

Lemma 3.8. $D$ is trivial iff $G(D)$ is trivial.

Theorem 3.9. Suppose $D$ is 1-based. Then either $D$ is trivial, or the geometry of $G(D)$ is projective geometry over a division ring.

Proof. Assume that $D$ is nontrivial. By the above lemma and Fact 1.5, it suffices to show that any independent pair $(a, b) \in G(D)$ (that is, $\operatorname{dim}(a b)=2$ ) is nontrivial in $G(D)$ (i.e., there is $c \in \operatorname{cl}(a b) \backslash(\operatorname{cl}(a) \cup \operatorname{cl}(b))$ where $c l$ is the closure of $G(D))$.

Claim 1. Any independent pair $(c, d) \in D$ is nontrivial in $G(D)$. Indeed, since $G(D)$ is modular, if there is an independent pair from $D$ trivial in $G(D)$, then any independent pair from $D$ is trivial in $G(D)$. (Otherwise, by type amalgamation one can find a non-modular triangle that violates modularity of $G(D)$. See the proof of 1.8.) Then any independent triple $(a, b, c) \in D$ is also trivial in $G(D)$ (otherwise, say $d \in \operatorname{cl}(a b c) \backslash(\operatorname{cl}(a) \cup \operatorname{cl}(b) \cup \operatorname{cl}(c))$, then $a b$ and $c d$ violate modularity of $G(D))$. Iterating this argument shows that $D$ is trivial, which is not the case.

Claim 2. Any independent pair $(a, b) \in G(D)$ with $a \in D$ is nontrivial in $G(D)$.

Suppose not, say the independent pair $(a, b) \in G(D)$ with $a \in D$ is trivial. Now since $b \in G(D)$, there is an independent tuple $\bar{c}=\left(c_{1}, \ldots, c_{n}\right) \in D$ such that $b \in \operatorname{cl}\left(c_{1}, \ldots, c_{n}\right)$. By Claim 1, we can assume $n>1$. We can further assume that any $n-1$ subtuple of $\bar{c}$ is independent from $b$, and $\left\{a, b, c_{1}, \ldots, c_{n-1}\right\}$ is independent. Hence

$$
\operatorname{dim}(a, b, \bar{c})=n+1 .
$$

By Claim 1, there is $d \in \operatorname{cl}\left(a c_{1}\right) \backslash \operatorname{cl}(a) \cup \operatorname{cl}\left(c_{1}\right)$. It follows that $d \notin \operatorname{cl}\left(c_{2}, \ldots, c_{n}\right)$ (otherwise $a \in \operatorname{cl}(\bar{c})$, violating $(*))$. Hence the dimension of the set $B=\left\{d, c_{2}, \ldots, c_{n}\right\}$ is $n$, whereas by $\left(^{*}\right)$ and triviality of $(a, b)$, it can easily be seen that $c l(B) \cap c l(a b)=\emptyset$. However, by modularity of $G(D)$, this contradicts $\left(^{*}\right)$. Hence Claim 2 follows.

Claim 3. Any independent pair $(d, e) \in G(D)$ is nontrivial.

Suppose not, say the independent pair $(d, e) \in G(D)$ is trivial. There is a point $c \in D$ that is independent from $(d, e)$. By Claim $2,(c, d)$ and $(c, e)$ are nontrivial. Again this violates modularity of $G(D)$, since $(c, d, e)$ forms a non-modular triangle. This finishes the proof of Claim 3 and the theorem. 
The theorem shows Vassiliev's result cheaply. Namely, the geometry of 1-based $D$ is a subgeometry of projective geometry over a division ring.

From now on $D$ will be 1-based.

The (pre)geometry of $G(D)$ is not definable (it is a union of definable sets). However, with the reducibility condition we will discuss below, $G(D)$ can be dealt with as a definable object, at least for the $\omega$-categorical case.

Definition 3.10. We say $G(D)$ is reducible into (or has a reduction in) $D^{k}$ if for any point $a \in G(D)$ there is a $k$-tuple $\bar{b} \in D$ such that $a \in \operatorname{acl}(\bar{b})$.

When $D$ is locally modular, $G(D)$ has a reduction in $D^{2}$. In fact, more is true.

Lemma 3.11 ( $D$ locally modular). For $c \in G(D)$ and any $d \in D$, there is $e \in D$ such that $c \in$ acl $(d e)$.

Proof. By Claim 1 in the proof of Fact 3.2, there is $\bar{a}=\left(a_{1}, \ldots, a_{n}\right) \in D$ such that $\operatorname{acl}(\bar{a} d)=\operatorname{acl}(d c)$. If $\bar{a} \in \operatorname{acl}(d)$, then $c \in \operatorname{acl}(d)$, and we are done. Hence we may assume that some $a_{i}$, say $a_{1}$, is not in $\operatorname{acl}(d)$. Then, since $r k\left(a_{1}\right)=1, a_{1}$ forks with $c$ over $d$. By transitivity, $c$ forks with $a_{1} d$. Since $r k(c)=1, c \in \operatorname{acl}\left(d a_{1}\right)$.

Now, as by-products, we have the following lemmas.

Lemma 3.12. Assume that $D$ is nontrivial locally modular, and $p \in D$. The geometry of $D_{p}$ and the geometry of $G(D)$ are isomorphic as projective geometries.

Proof. Since $G(D)^{\prime}$, the geometry of $G(D)$, is that of infinite-dimensional projective space over some ring, $G(D)_{p}^{\prime} \cong G(D)^{\prime}$. Now since $D \subseteq G(D)$, clearly $D_{p}$ is a subpregeometry of $G(D)_{p}$. Also, by the previous lemma, given $x \in G(D) \backslash \operatorname{cl}(p)$, there is $e \in D$ such that $c l(p x)=\operatorname{cl}(e x)$ in $G(D)$. Hence $D_{p}^{\prime} \cong G(D)_{p}^{\prime} \cong G(D)^{\prime}$, and the lemma holds.

Lemma 3.13 ( $D$ locally modular). If $G(D)^{\prime}$ is projective over a field with $\operatorname{Card}(F)$ $\leq 3$, then $D^{\prime}$, the geometry of $D$, is affine or projective.

Proof. This can be shown by checking the possible cases, using amalgamation and 3.11 , working in a projective plane. Let us first prove this when $\operatorname{Card}(F)=3$.

Claim 1. There is no independent $\{a, b\} \subseteq D^{\prime}$ such that $c l(a b) \cap D^{\prime}=\{a, b\}$. Indeed, if there exist such $a, b$, then by amalgamation we can find $c \in D^{\prime}$ such that $\operatorname{dim}(a b c)=3$ and any closed set in $D^{\prime}$ generated by a 2 -subset of $\{a, b, c\}$ is itself. Let $b^{\prime} \in\left(c l(b c) \cap G(D)^{\prime}\right) \backslash D^{\prime}$. Then by 3.11, we can find $d \in D^{\prime}$ such that $b^{\prime} \in \operatorname{cl}(a d)$. By modularity and the fact that any line in $G(D)^{\prime}$ has size 4 , there are $c^{\prime} \in \operatorname{cl}(a c) \cap \operatorname{cl}(b d) \cap G(D)$ and $c^{\prime \prime} \in \operatorname{cl}(a c) \backslash D^{\prime}$ such that $c^{\prime \prime} \neq a, c, c^{\prime}$. Then it is easy to see that $a, b, c \notin c l\left(d c^{\prime \prime}\right)$. Hence $\emptyset \neq c l\left(d c^{\prime \prime}\right) \cap c l(b c) \in G(D) \backslash D$, and also $c l\left(d c^{\prime \prime}\right) \cap \operatorname{cl}(a b) \in G(D) \backslash D$. Therefore $c l\left(d c^{\prime \prime}\right) \cap D^{\prime}=\{d\}$, which contradicts 3.11. Hence Claim 1 is proved.

Claim 2. There cannot exist independent pairs $\left\{a_{1}, a_{2}\right\},\left\{b_{1}, b_{2}\right\} \subseteq D^{\prime}$ such that $\operatorname{Card}\left(c l\left(a_{1} a_{2}\right) \cap D^{\prime}\right)=3$ and $\operatorname{Card}\left(c l\left(b_{1} b_{2}\right) \cap D^{\prime}\right)=4$.

Suppose that there are such points $a_{i}$ and $b_{i}$ in $D^{\prime}$. By amalgamation, there are $\{u, v, w\} \subseteq D^{\prime}$ such that

$$
\operatorname{Card}\left(\operatorname{cl}(u v) \cap D^{\prime}\right)=\operatorname{Card}\left(\operatorname{cl}(v w) \cap D^{\prime}\right)=3, \quad \operatorname{Card}\left(c l(u w) \cap D^{\prime}\right)=4
$$

and

$$
\operatorname{dim}(u v w)=3 .
$$


Choose $v^{\prime} \in \operatorname{cl}(u v) \backslash D^{\prime}$ and $w^{\prime} \in \operatorname{cl}(v w) \backslash D^{\prime} v^{\prime}$. Then, since $\operatorname{Card}\left(\operatorname{cl}(u w) \cap D^{\prime}\right)=4$, by modularity there is $z \in \operatorname{cl}(u w) \cap \operatorname{cl}\left(v^{\prime} w^{\prime}\right) \cap D^{\prime}$. Then $1 \leq \operatorname{Card}\left(\operatorname{cl}\left(v^{\prime} w^{\prime}\right) \cap D^{\prime}\right) \leq 2$, contradicting 3.11 and Claim 1. Hence Claim 2 follows, and the lemma is established for $\operatorname{Card}(F)=3$ by Fact 1.6.

When $\operatorname{Card}(F)=2$, by a similar argument, it can be seen that one cannot amalgamate independent $\left(c_{1} c_{2}\right),\left(c_{1} c_{2}\right)$ and $\left(d_{1} d_{2}\right)$ from $D^{\prime}$ such that

$$
\operatorname{Card}\left(c l\left(c_{1} c_{2}\right) \cap D^{\prime}\right)=2 \text { and } \operatorname{Card}\left(\operatorname{cl}\left(d_{1} d_{2}\right) \cap D^{\prime}\right)=3 .
$$

Then 1.6 says that the lemma holds for this case as well.

Remark 3.14. If $D$ is a generic subset of locally modular $U$, then $G(D)$ also has a reduction in $D^{2}$. Indeed, let $c \in G(D)$. Then by 3.11, there are $d \in D, e \in U$ such that $c \in \operatorname{acl}(d e)$. Now there exists $(a, b)$ realizing $t p(d e / c)$ such that

$$
a b \underset{c}{\downarrow} d e .
$$

Then, by the remark in the proof of 3.2, $a c l(g)=a c l(c)$ where $g=c b(a b / d e)$. By the genericity of $D$, there exists $\left(a^{\prime}, b^{\prime}\right) \in D$ such that $t p^{-}(a b / d e)=t p^{-}\left(a^{\prime} b^{\prime} / d e\right)$ in the language of $U$ without the predicate $D$. Then $a^{\prime} b^{\prime}$ preserves the canonical base of $t p(a b / d e)$. Hence $a c l(c)=\operatorname{acl}(g) \subseteq \operatorname{acl}\left(a^{\prime} b^{\prime}\right)$. We are done. (In this case, of course, strong reducibility in $D^{2}$ as in Lemma 3.11 does not follow.)

In general, the following holds. Hence any $G(D)$ is reducible into $D^{3}$ for 1-based D.

Theorem 3.15. Let $u \in G(D)$.

(1) Suppose that $(x, y)$ is a fixed independent pair from $D$. Then $u \in \operatorname{acl}\left(x^{\prime} y^{\prime} z\right)$ where $x^{\prime}, y^{\prime}, z \in D$ and $t p(x y)=t p\left(x^{\prime} y^{\prime}\right)$.

(2) Suppose that there are independent $\{x, y\}$ from $D$ such that for any $z \in$ $\operatorname{acl}(x y) \cap G(D), z \in \operatorname{acl}\left(z^{\prime}\right)$ for some $z^{\prime} \in(\operatorname{acl}(x y) \cap D)$, i.e., a full line is already in $D$. Then $G(D)$ is reducible into $D^{2}$.

(3) For given $d_{1} \in D$, there is $d_{2} d_{3} \in D$ such that $u \in \operatorname{acl}\left(d_{1} d_{2} d_{3}\right)$.

Proof. We first prove (1) using induction on $n$ where $u \in G(D)$ has a reduction in $D^{n}$. The case $n=0$ (that is, $u \in \operatorname{acl}(\emptyset)$ ) is trivial. Now suppose that $u \in \operatorname{acl}\left(a_{1} \ldots a_{n+1}\right) \quad\left(a_{i} \in D\right)$ with the induction hypothesis for $n$. We will verify (1) for $n+1$. We can assume that $\left\{a_{1}, \ldots, a_{n+1}\right\}$ is independent. Now let $g=c b\left(u a_{1} / a_{2} \ldots a_{n+1}\right)$. Thus $S U(g)=1$ and $g \in G(D)$. Since $a_{1} \notin a c l(g) \subseteq$ $\operatorname{acl}\left(a_{2} \ldots a_{n+1}\right)$,

$$
u \text { is in the line generated by }\left\{g, a_{1}\right\} \text {. }
$$

Then, since $g \in \operatorname{acl}\left(a_{2} \ldots a_{n+1}\right)$, by the induction hypothesis, $g \in \operatorname{acl}(b c d)$ for some $c, d \in D$ such that $t p(c d)=t p(x y)$. If $g$ is already in $a c l(c d)$, then, by $(*), u \in$ $\operatorname{acl}\left(g a_{1}\right) \subseteq \operatorname{acl}\left(c d a_{1}\right)$. Thus, in this case, the theorem holds. Therefore we only need to consider the case when $g \notin a c l(c d)$. We can also clearly assume that $g \notin \operatorname{acl}(b)$ (otherwise we are done). Then, since $G(D)$ is modular, we can find $v \in \operatorname{acl}(c d) \cap \operatorname{acl}(b g) \cap G(D)$ such that

$$
g \in \operatorname{acl}(b v)
$$

and $\operatorname{dim}(v g)=2$. Also,

$(* *) \quad$ at least one of $\{v, c\}$ or $\{v, d\}$ (say $\{v, d\}$ ) is independent. 
Now, since $d, a_{1} \in D, \operatorname{Lstp}(d)=\operatorname{Lstp}\left(a_{1}\right)$. Hence, we can find $v^{\prime}$ such that

$$
\operatorname{Lstp}(v d)=\operatorname{Lstp}\left(v^{\prime} a_{1}\right) \text {. }
$$

In particular, $\operatorname{Lstp}(v)=\operatorname{Lstp}\left(v^{\prime}\right)$. Then by $(*),(* *),(\star)$, we can amalgamate types $t p\left(v^{\prime} / a_{1}\right)$ and $t p(v / g)$, so that we obtain $v^{\prime \prime} \models t p\left(v^{\prime} / a_{1}\right) \cup t p(v / g)$. Hence, there are $b^{\prime}, c^{\prime}$ such that $t p(v g b)=t p\left(v^{\prime \prime} g b^{\prime}\right), t p(v d c)=t p\left(v^{\prime \prime} a_{1} c^{\prime}\right)($ by $(\star))$, and so by $(\dagger)$,

$$
t p\left(c^{\prime} a_{1}\right)=t p(x y), v^{\prime \prime} \in \operatorname{acl}\left(c^{\prime} a_{1}\right) \text { and } g \in \operatorname{acl}\left(b^{\prime} v^{\prime \prime}\right) .
$$

Then by $(*),(\ddagger)$,

$$
u \in \operatorname{acl}\left(g a_{1}\right) \subseteq \operatorname{acl}\left(g a_{1} v^{\prime \prime}\right) \subseteq \operatorname{acl}\left(a_{1} b^{\prime} v^{\prime \prime}\right) \subseteq \operatorname{acl}\left(c^{\prime} a_{1} b^{\prime}\right) .
$$

Since $t p\left(c^{\prime} a_{1}\right)=t p(x y)$, the $(n+1)$-th induction hypothesis for $(1)$ is deduced. Therefore we have proved (1).

(2) comes from (1) and the modularity of $G(D)$.

For (3), use the proof of (1), replacing $g$ with $u$ and $a_{1}$ with $d_{1}$.

Remark 3.16. From the above theorem 3.15, we can identify any element $u$ in $G(D)$ as $e=\bar{d} / E \in G(D)$ (i.e., $\operatorname{acl}(u)=\operatorname{acl}(e))$ where $\bar{d} \in D^{6}$ and $E$ is a definable equivalence relation on $D^{6}$ (over $\operatorname{dom}(D)$ ), as follows: As usual we take $\operatorname{dom}(D)=\emptyset$ (by naming $\operatorname{dom}(D)$ ) for notational simplicity. By 3.15 , there are triples $(a, b, c),\left(a^{\prime}, b^{\prime}, c^{\prime}\right) \in D^{3}$ such that $u \in a c l(a b c),\left(a^{\prime} b^{\prime} c^{\prime}\right) \models t p(a b c / u)$ and

$$
a b c \underset{u}{\downarrow} a^{\prime} b^{\prime} c^{\prime} .
$$

Then, by the remark in 3.2 ,

$$
\operatorname{acl}(u)=\operatorname{acl}(g)
$$

where $g=c b\left(a b c / a^{\prime} b^{\prime} c^{\prime}\right)$. Now, if an automorphism fixes $\left(a b c a^{\prime} b^{\prime} c^{\prime}\right)$, then, since the parallelism class of $L s t p\left(a b c / a^{\prime} b^{\prime} c^{\prime}\right)$ is setwise fixed, so is $c b\left(a b c / a^{\prime} b^{\prime} c^{\prime}\right)$, i.e., $g \in d c l\left(a b c a^{\prime} b^{\prime} c^{\prime}\right)$. Hence we can identify $g=\left(a b c a^{\prime} b^{\prime} c^{\prime}\right) / \bar{F}$ where $\bar{F}$ is a typedefinable equivalence relation on $D^{6}$. Therefore, since $S U(g)=1$, as in 3.5.2, there is a definable equivalence relation $E \in \bar{F}$ such that $\left(a b c a^{\prime} b^{\prime} c^{\prime}\right) / E$ in $G(D)$ and $g$ are interalgebraic. Since $u$ and $g$ are interalgebraic, the result follows.

In particular, if $D$ is $\omega$-categorical, then, since there are only finitely many types and equivalence relations over $\operatorname{dom}(D)$ on $D^{6}$, by the above remark, the geometry of $G(D)$ is isomorphic to that of some union of finitely many definable sets in finitely many sorts in $G(D)$. Since the union itself is $\omega$-categorical even as a 1-sorted structure, we have the following results.

Corollary 3.17. Let $D$ be $\omega$-categorical. Then, $D$ is trivial or the geometry of $G(D)$ is projective over a finite field.

Definition 3.18. Let $G(D)$ have a reduction in $D^{k}$ where $k$ is minimal such. (Hence $k \leq 3$.) By a hypersurface of $D^{k}$, we mean a $L s t p\left(a_{1} \ldots a_{k} / A\right)$ of rank $k-1$ $\left(a_{i} \in D\right)$. We call a hypersurface of $D^{k}$ with rank-0 canonical base rigid.

The projective closure of $D$, denoted $P(D)$, is defined as

$$
P(D)=D \cup\left\{e \mid e \text { is the canonical base of a non-rigid hypersurface of } D^{k}\right\} \text {. }
$$

By a rank calculation, the rank of the canonical base of a hypersurface of $D^{k}$ is $\leq 1$. Hence $P(D)$ is a collection of canonical bases $e=c b\left(a_{1} \ldots a_{k} / e\right)$ where $S U\left(a_{1} \ldots a_{k} / e\right)=k-1$ for independent $\left(a_{1}, \ldots, a_{k}\right) \in D^{k}$. Since $S U(e)=1$, by 
Remark 3.5.2 we can assume that $P(D) \subseteq G(D)$ by identifying $e$ with some interalgebraic $g \in G(D)$. If $D$ is $\omega$-categorical, we can moreover assume, as in Remark 3.16 , that $P(D)$ is a dom $(D)$-definable subset of $G(D)$.

Lemma 3.19 (Notation as in 3.16). For any point $u \in G(D)$, there is $g \in P(D)$ such that $g$ and $u$ are interalgebraic. (Hence the geometries of $P(D)$ and $G(D)$ coincide.)

Proof. This is immediate from Remark 3.16, since there $S U\left(a b c / a^{\prime} b^{\prime} c^{\prime}\right)=2$.

Then we get the following theorem immediately. This answers the main conjecture in 21] positively.

Theorem 3.20. Suppose $D$ is $\omega$-categorical. Then $P(D)$ is $\operatorname{dom}(D)$-definable (in $\left.D^{e q}\right)$, as is its geometry $P(D)^{\prime}$. Hence, if $D$ is nontrivial, $P(D)^{\prime}$ has a strongly minimal stable reduct (over $\operatorname{dom}(D))$ preserving the projective geometry over a finite field $F$. Hence, over a finite parameter in $D^{e q}$, $D$ interprets a vector space (as projective and affine spaces do) over $F$.

(Recently Ben-Yaacov, Tomasic and Wagner obtained an almost hyperimaginary group configuration in 2]. Using this, they also proved the interpretability of a vector space in an $\omega$-categorical nontrivial $D[20]$.)

Now we study the relationship between the whole theory and rank-1 types. As in the stable case, the notion of triviality can be imposed on the underlying theory.

Definition 3.21. We say that $T$ is trivial if for $a, b, c, A \subseteq \bar{M}^{e q}$, whenever $\{a, b, c\}$ is pairwise independent over $A$, then $\{a, b, c\}$ is independent over $A$.

By mimicking the proofs in the stable case found in [17, 4.2.5], with some novel argument, we can show Lemma 3.22 below. The following easy fact will be used. In any simple $T$, if a finite tuple $\bar{a}$ is not in $\operatorname{acl}(\emptyset)$, then there is a complete extension $p$ of $t p(\bar{a})$ such that $S U(p)=1$. Indeed, let Let $t p(\bar{a})=p_{o} \subseteq p_{1} \ldots \subseteq p_{i} \ldots$ be a maximal non-algebraic forking chain. (The length is at most $|T|$.) Then $p=\bigcup_{i} p_{i}$ is non-algebraic as well, and, by maximality, $S U(p)=1$.

Lemma 3.22. Suppose that $T$ is 1-based. Then $T$ is trivial if and only if all rank-1 types are trivial.

Proof. We only need to prove the right to left direction, since the other direction is obvious. Suppose that there exists a nontrivial triple $(a, b, c)$ over $A$, i.e., $(a, b, c)$ is pairwise independent over $A$, whereas $\{a, b, c\}$ is dependent over $A$. We will find a nontrivial rank-1 type.

Claim 1. We may assume that $a \in \operatorname{acl}(b c A), b \in \operatorname{acl}(a c A)$ and $c \in \operatorname{acl}(b a A)$ (but $\{a, b, c\}$ is still pairwise independent over $A)$.

Indeed, if we choose

$$
a_{0} \in \operatorname{cb}(a / b c A) \backslash \operatorname{acl}(A), \quad b_{0} \in \operatorname{cb}\left(b / a_{0} c A\right) \backslash a c l(A), \quad c_{0} \in \operatorname{cb}\left(c / a_{0} b_{0} A\right) \backslash a c l(A)
$$

such that $c b(a / b c A) \subset \operatorname{acl}\left(a_{0}\right)$ and similarly for $b_{0}, c_{0}$, then, by 1-basedness, these elements will suffice.

Now, for notational convenience, let $A=\emptyset$.

Claim 2. There is $F$ such that $S U(a / F)=S U(b / F)=S U(c / F)=1$ and $(a, b, c)$ is still a nontrivial triple over $F$. 
Indeed, by the remark before 3.22 , there is $d$ such that $S U(a / d)=1$. We assume $d=c b(a / d)$. Then, by 1-basedness, $d \in \operatorname{acl}(a)$ and

$$
b d \underset{F}{\downarrow} c d
$$

where $F=a c l(b d) \cap a c l(c d)$. Then

$$
b \underset{F}{\downarrow} c .
$$

Now we have $S U(a / F)=1$, since otherwise $a \in \operatorname{acl}(b d)$. So $a \in \operatorname{acl}(d)$

$$
\text { (as } a d \underset{\emptyset}{\downarrow} \quad b)
$$

contradicting the fact that $S U(a / d)=1$. Similarly,

$$
a \underset{F}{\downarrow} b
$$

and

$$
a \underset{F}{\downarrow} c .
$$

Now, by the rank calculation of $S U(a b / c F)$,

$$
S U(b / c F)=S U(b / F)=1,
$$

since $S U(a / c F)=1$. Similarly, by the rank calculation of $S U(a c / b F)$,

$$
S U(c / b F)=S U(c / F)=1 .
$$

Hence, Claim 2 is established.

Claim 3. $t p(a / F)$ is nontrivial. (This finishes the proof.)

First, there are $a_{1}, a_{2}, b^{\prime}, c^{\prime}$ such that

$$
t p\left(a_{1} c^{\prime} / F b\right)=t p(a c / F b), \quad c^{\prime} \underset{F}{\downarrow} a b c, t p\left(a_{2} b^{\prime} / F c\right)=t p(a b / F c)
$$

and

$$
b^{\prime} \underset{F}{\downarrow} a b c c^{\prime} .
$$

Hence

$$
b^{\prime} c^{\prime} \underset{F}{\downarrow} a b c .
$$

Now we subclaim that $a, a_{1}, a_{2}$, all realizing the rank- 1 type $t p(a / F)$, form a nontrivial triple over $F b^{\prime} c^{\prime}$. For pairwise independence, we only show that

$$
a_{1} \underset{F b^{\prime} c^{\prime}}{\downarrow} a_{2} ;
$$

the other cases follow similarly. We have

$$
b \underset{F}{\downarrow} c ;
$$

hence

$$
b \underset{F b^{\prime} c^{\prime}}{\downarrow} c
$$


by $(* *)$. Thus

$$
F b c^{\prime} \underset{F b^{\prime} c^{\prime}}{\downarrow} F c b^{\prime} .
$$

Then

$$
a_{1} \underset{F b^{\prime} c^{\prime}}{\downarrow} a_{2}
$$

by Claim 2 and $(*)$. Now since $a \in \operatorname{acl}(b c)$, again by $\left(^{*}\right)$ and Claim 2, each of $a, a_{1}, a_{2}$ is algebraic over $F b^{\prime} c^{\prime}$ together with the other two. Hence the subclaim is verified.

Secondly, by 1-basedness, there is a tuple $a_{3} a_{4} a_{5}$ realizing $\operatorname{tp}\left(a a_{1} a_{2} / F b^{\prime} c^{\prime}\right)$ such that

$$
a a_{1} a_{2} \underset{a_{3} a_{4} a_{5}}{\downarrow} F b^{\prime} c^{\prime} \text { and } a a_{1} a_{2} \underset{F b^{\prime} c^{\prime}}{\downarrow} a_{3} a_{4} a_{5} .
$$

Then, it can be easily seen that $\left(a, a_{1}, a_{2}\right)$ forms a nontrivial triple over $F a_{3} a_{4} a_{5}$. This means that the localized geometry of $t p(a / F)$ at $\left\{a_{3}, a_{4}, a_{5}\right\}$ is nontrivial, since all the $a$ and $a_{i}$ realize the rank-1 type $\operatorname{tp}(a / F)$. Hence, the original type $\operatorname{tp}(a / F)$ cannot be trivial either. We have proved Claim 3 and the lemma.

Recall that if $T$ is 1-based, then for any $A$ and tuple $\bar{a}$, there is $\bar{a}^{\prime}$ with $|\bar{a}|=\left|\bar{a}^{\prime}\right|$ such that $D(t p(\bar{a} / A), \varphi, k)=D\left(t p\left(\bar{a} / \bar{a}^{\prime}\right), \varphi, k\right)$, for each $\varphi, k$ (by taking an element in a Morley sequence, for example). Thus, if additionally $T$ is $\omega$-categorical, then $T$ is supersimple, since every forking chain must have finite length. Moreover, since $S U(\bar{a} / A)=S U\left(\bar{a} / \bar{a}^{\prime}\right)$, any $n$-type in $T$ has finite $S U$-rank ([9 4.7$]$ ). (Evans and Wagner [8] showed that any supersimple, $\omega$-categorical group or any CM-trivial theory also has finite $S U$-rank.)

By previous results in this section, we obtain the following.

Corollary 3.23. Let $T$ be nontrivial, 1 -based and $\omega$-categorical. Then an infinitedimensional vector space over a finite field, in particular the infinite additive group, is definable in $\bar{M}^{\text {eq }}$ over a finite parameter.

\section{REFERENCES}

[1] E. Artin, Geometric algebra, Interscience Publishers, New York (1957). MR 18:553e

[2] I. Ben-Yaacov, I. Tomasic and F. O. Wagner, Constructing an almost hyperdefinable group, preprint, 2001.

[3] S. Buechler, The geometry of weakly minimal types, J. of Symbolic Logic 50 (1985) 1044-1053. MR 87k:03029

[4] S. Buechler, A. Pillay and F. O. Wagner Supersimple theories, J. Amer. Math. Soc. 14 (2001) 109-124. MR 2001j:03065

[5] Z. Chatzidakis and A. Pillay, Generic structures and simple theories, Ann. of Pure and Applied Logic 95 (1998) 71-92. MR 2000c:03028

[6] G. Cherlin, L. Harrington, and A. H. Lachlan, $\aleph_{0}$-categorical, $\aleph_{0}$-stable structures, Ann. Pure and Applied Logic 28 (1985) 103-135. MR 86g:03054

[7] J. Doyen and X. Hubaut, Finite regular locally projective spaces, Math. Z. 119 (1971) 83-88. MR 43:3897

[8] D. Evans and F. O. Wagner, Supersimple $\omega$-categorical groups, J. of Symbolic Logic 65 (2000) 767-776. MR 2001f:03067

[9] B. Hart, B. Kim and A. Pillay, Coordinatisation and canonical bases in simple theories, Journal of Symbolic Logic, 65 (2000) 293-309. MR 2001j:03066

[10] E. Hrushovski, Unimodular minimal theories, Journal of London Math. Soc., 46 (1992) 385396. MR 94b:03062

[11] E. Hrushovski, Locally modular regular types, in Classification theory, Lecture Notes in Math. 1292, Springer-Verlag, Berlin (1987) 132-164. MR 90n:03064 
[12] E. Hrushovski, The Mordell-Lang conjecture for function fields, J. Amer. Math. Soc. 9 (1996) 667-690. MR 97h:11154

[13] E. Hrushovski, Simplicity and the Lascar group, preprint (1998).

[14] E. Hrushovski and S. Shelah, A dichotomy theorem for regular types, Ann. of Pure and Applied Logic 45 (1989) 157-169. MR 91g:03068

[15] B. Kim, Forking in simple unstable theories, Journal of London Math. Soc. (2) 57 (1998) 257-267. MR 2000a:03052

[16] B. Kim and A. Pillay, Simple theories, Ann. of Pure and Applied Logic 88 (1997) 149-164. MR 99b:03049

[17] A. Pillay, Geometric stability theory, Oxford University Press, Oxford (1996). MR 98a:03049

[18] S. Shelah, Simple unstable theories, Annals of Math. Logic 19 (1980), 177-203. MR 82g:03055

[19] S. Shelah, Classification theory, 2nd edition, North-Holland, Amsterdam (1990). MR 91k:03085

[20] I. Tomasic and F. O. Wagner, Applications of the group configuration theorems in simple theories, preprint, 2001.

[21] E. Vassiliev, Expansions of rank 1 structures, Ph. D. Thesis, Univ. of Notre Dame (2001).

[22] F. O. Wagner, Simple theories, Kluwer Academic Publishers, Dordrecht (2000). MR 2001b:03035

[23] B. I. Zilber, Uncountably categorical theories, Amer. Math. Soc. Translations of Mathematical Monographs, Vol. 117 (1993). MR 94h:03059

Mathematics Department, Massachusetts Institute of Technology, 77 Massachusetts Ave., Cambridge, Massachusetts 02139

E-mail address: tdpdp@math.mit.edu

Mathematics Department, Massachusetts Institute of Technology, 77 Massachusetts Ave., Cambridge, Massachusetts 02139

E-mail address: bkim@math.mit.edu 